\section{Pacific Northwest}

National Laboratory

Operated by Battelle for the

U.S. Department of Energy

\title{
Progress Report for the Advanced Large-Area Plastic Scintillator (ALPS) Project: FY 2003 Final
}

\author{
P. L. Reeder \\ R. C. Craig \\ D.L. Stephens, Jr. \\ B. Geelhood \\ D.V. Jordan
}

November 2003

\footnotetext{
Prepared for the U.S. Department of Energy
} under Contract DE-AC06-76RL01830 


\title{
DISCLAIMER
}

This report was prepared as an account of work sponsored by an agency of the United States Government. Neither the United States Government nor any agency thereof, nor Battelle Memorial Institute, nor any of their employees, makes any warranty, express or implied, or assumes any legal liability or responsibility for the accuracy, completeness, or usefulness of any information, apparatus, product, or process disclosed, or represents that its use would not infringe privately owned rights. Reference herein to any specific commercial product, process, or service by trade name, trademark, manufacturer, or otherwise does not necessarily constitute or imply its endorsement, recommendation, or favoring by the United States Government or any agency thereof, or Battelle Memorial Institute. The views and opinions of authors expressed herein do not necessarily state or reflect those of the United States Government or any agency thereof.

\author{
PACIFIC NORTHWEST NATIONAL LABORATORY \\ operated by \\ BATTELLE \\ for the \\ UNITED STATES DEPARTMENT OF ENERGY \\ under Contract DE-ACO6-76RLO183O
}

This document was printed on recycled paper. 


\section{Progress Report for the Advanced Large-Area Plastic Scintillator (ALPS) Project: FY 2003 Final}

November 2003
P. L. Reeder
D. L. Stephens, Jr.
D. V. Jordan
R. C. Craig
B. Geelhood

Prepared for the U.S. Department of Energy under Contract DE-AC06-76RL01830

Pacific Northwest National Laboratory Richland, Washington 99352 


\section{Summary}

Pacific Northwest National Laboratory is investigating possible technological avenues for substantially advancing the state-of-the-art in gamma detection via large-area plastic scintillators. This report describes progress on this project as of the conclusion of FY 2003. The primary focus of the report is on experimental tests conducted with a single large-area plastic scintillator outfitted with a variety of photomultiplier tube configurations. Measurements performed to date include scintillator response under broad-area exposure to various point-like gamma sources, and light-output uniformity mappings obtained by varying the position of a collimated beta-source over the surface of the scintillator. Development of a Monte Carlo program for modeling the response of a large-area scintillator sensor to ionizing radiation, explicitly including resolution-broadening effects of scintillation light generation, propagation, and collection is also described. 


\section{Acknowledgements}

The authors would like to thank W.K. Pitts and G. Dudder for many helpful discussions over the course of this project. Support for this project by the National Nuclear Security Administration's Office of Nonproliferation Research and Engineering, NA-22, is gratefully acknowledged. Pacific Northwest National Laboratory is operated for the U.S. Department of Energy by Battelle under Contract DE-AC0676RLO 1830. 


\section{Acronyms}

$\begin{array}{ll}\text { ADC } & \text { analog-to-digital converter } \\ \text { ALPS } & \text { Advanced Large-Area Plastic Scintillators } \\ \text { CAMAC } & \text { Computer Automated Measurement and Control } \\ \text { CERN } & \text { Center for European Nuclear Research } \\ \text { CPU } & \text { central processing unit } \\ \text { FWHM } & \text { full width at half maximum } \\ \text { NIM } & \text { Nuclear Instrument Module } \\ \text { PE } & \text { photoelectron } \\ \text { PMT } & \text { photomultiplier tube } \\ \text { TDC } & \text { Time to Digital (converter) } \\ \text { QDC } & \text { Charge to Digital (converter) } \\ \text { QE } & \text { quantum efficiency } \\ \text { ROI } & \text { region of interest } \\ \text { SCSI } & \text { small computer systems interface }\end{array}$




\section{Contents}

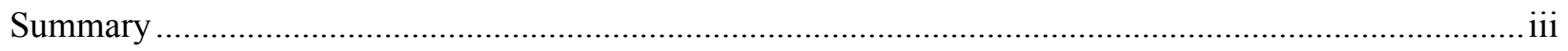

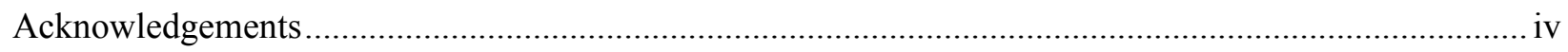

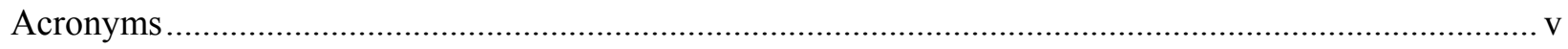

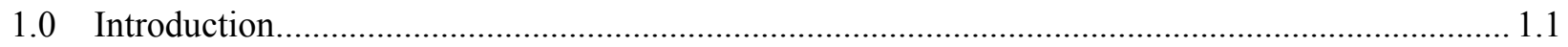

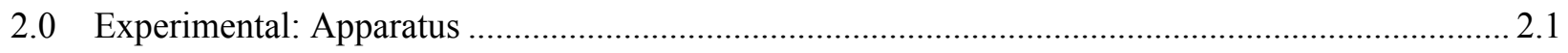

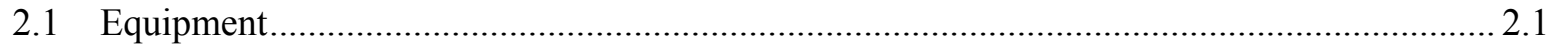

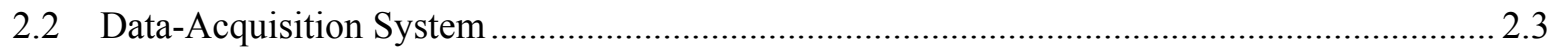

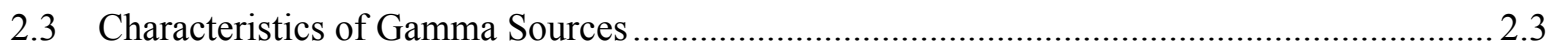

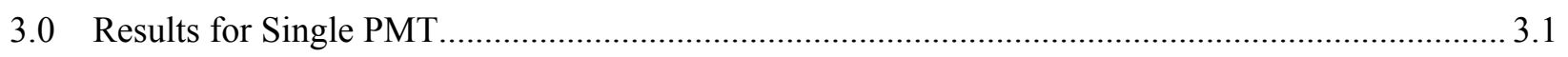

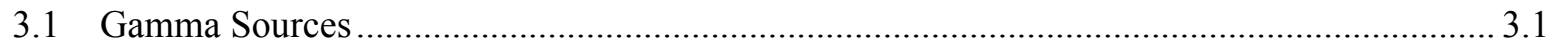

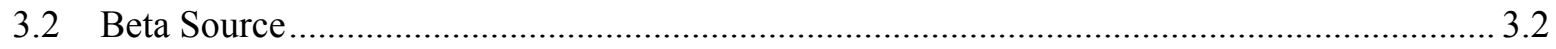

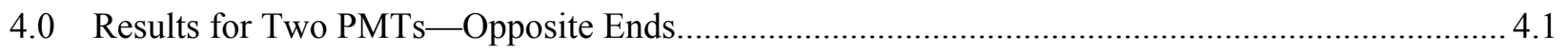

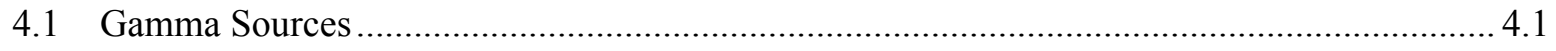

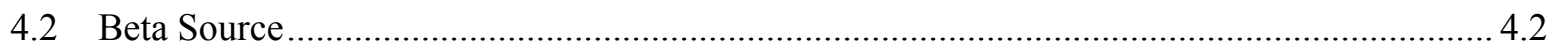

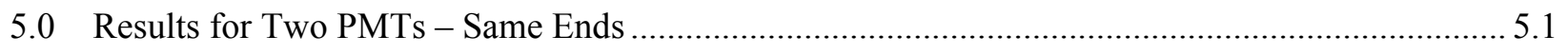

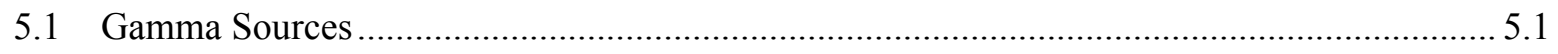

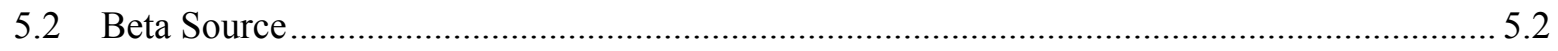

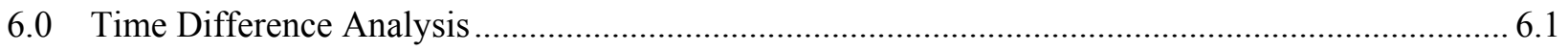

6.1 Calibration of Location Versus Time Difference ........................................................... 6.1

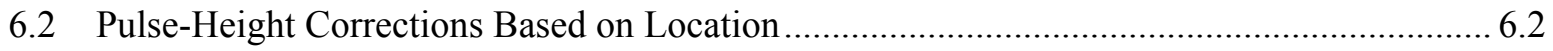

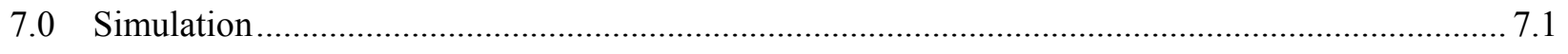

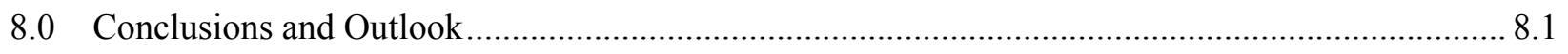

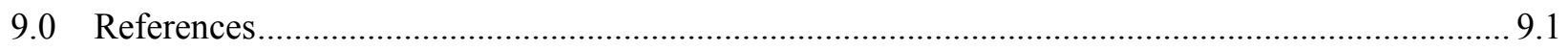

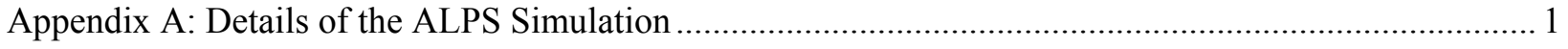


Appendix B: Measurements on 30-cm $\times 30-\mathrm{cm}$ Beveled Corner Scintillator

\section{Figures}

2.1. Design Drawing of the ALPS Project Apparatus Showing Two Scintillator Slabs with Three PMTs on each End of both Slabs.

2.2. Photographs of the ALPS Scintillator Sensor as Configured for Laboratory Tests at the Conclusion of FY 2003

3.1. Gamma Pulse Height Spectra for Single PMT Configuration

3.2. Beta Pulse Height Spectra from a Single PMT at Three Locations Along Mid-Point Axis

3.3. Mean Channel Number of Pulse Height Distributions for Beta Source at Various Distances from the Single PMT

4.1. Gamma Pulse Height Spectra for Sum of Pulses from each PMT for Configuration with a PMT on Opposite Ends

4.2. Mean Channel Number of Pulse Height Distributions for Beta Source at Various Distances Along the Mid-Point Axis Between Coincident PMTs at Opposite Ends of the Scintillator

4.3. Mean Channel Number of Pulse Height Distributions for Beta Source at Various Distances Along the Axis Parallel to the Mid-Point Axis But $17.8 \mathrm{~cm}$ away from it

5.1. Gamma Pulse Height Spectra for Configuration with two PMTs on same End

5.2. Mean Channel Number of Pulse Height Distributions Along Midpoint Axis for Configuration with two PMTs on the same End of the Plastic Scintillator

5.3. Mean Channel Number of Pulse Height Distributions Along Axis Parallel to Midpoint Axis at $-17.8 \mathrm{~cm}$ for Configuration with two PMTs on the same End of the Plastic Scintillator

6.1. Time-Difference Histograms of Two Beta Spectra Collected at 2.54-cm Separation on Scintillator.

6.2. Calibration Curve for Time Difference Versus Position for the Configuration with one PMT on each End of the Scintillator

7.1. Simulation Output for Various Gamma Sources in Single-PMT Configuration

7.2. Effect of Final Pulse-Smearing Event Processing Stage on Simulation Output, ${ }^{54} \mathrm{Mn}$ Gamma Source, One-PMT Configuration.

7.3. Comparison of Simulation to Experimental Data for ${ }^{54} \mathrm{Mn}$ Source in Single-PMT Scintillator Configuration.....

7.4. Comparison of Simulation to ${ }^{54} \mathrm{Mn}$ Source Data for Configuration with One PMT at each End.... 7.5 


\section{Tables}

2.1. Gamma Sources, Energies, Maximum Energy of Compton Scattered Electron, and Gamma Abundances for Sources Used in this Work 


\subsection{Introduction}

The primary goal of the Advanced Large-Area Plastic Scintillator (ALPS) project is to investigate the limits of gamma-ray detection using large area plastic scintillators, with particular emphasis on the use of plastic for detection of radiological threats in vehicle portal monitors. The current generation of portal monitors deployed at international border crossings relies heavily on large-area plastic scintillators for detecting gamma rays emitted by potentially hazardous, clandestinely transported radioactive materials. A large detector area is important for portal-monitor applications to maximize gamma-detection count rate, and thus source-detection sensitivity, because spectrum acquisition times are severely limited by the need to accommodate a reasonable flow of vehicles past the portal. Plastic scintillator is relatively inexpensive per unit area and rugged in comparison to other scintillating materials, e.g., NaI(Tl), and semiconductors, e.g., high-purity germanium, and thus represents an attractive material for constructing a large-area gamma sensor.

However, the limited energy-spectrum information available from a plastic scintillator presents challenges to reliably distinguishing radiological threats from naturally occurring and benign radioactive materials. The physical basis of particle detection with a scintillating material is the emission of light when ionizing radiation deposits energy in the material. Plastic scintillators typically emit about 10,000 light photons per $\mathrm{MeV}$ of deposited energy, about a factor of 4 fewer than in $\mathrm{NaI}(\mathrm{Tl})$. The intrinsic energy resolution of plastic, which scales as the square root of the number of information carriers, or light quanta in the case of scintillating material, is thus about a factor of 2 poorer than $\mathrm{NaI}(\mathrm{Tl})$. In addition to this inherent limitation on the number of information carriers generated, only a relatively small fraction of the scintillation light emitted in a typical large-area scintillator actually reaches the photomultiplier tubes (PMTs). In commercially available portal-monitor sensors with one or two 2-in.-diameter PMTs, the light collection efficiency can be as low as $3.5 \%$ (one tube) to $7 \%$ (two tubes). Thus, the net energy resolution of the scintillator can be as much as a factor of 5 poorer than the scintillator's intrinsic energy resolution. Light generation and collection limitations are not the only factors contributing to the relatively poor resolution of a plastic scintillator. Gammas interact in plastic primarily by Compton scattering rather than by photoelectric absorption. Thus, the full energy-deposition peak, or photopeak, a familiar feature of spectra in higher-Z materials such as $\mathrm{NaI}(\mathrm{Tl})$ and HPGe, is essentially absent in plastic scintillators of thicknesses typical to commercial units. Although energy-deposition information is available from the shape of the Compton scattering continuum in plastic, this information does not necessarily conform to the conventional gamma spectroscopist's expectation of well-defined, energy-localized peaks that represent a radioisotope's "fingerprint." These features of the spectroscopic response of plastic scintillator have conspired to render radionuclide identification in plastic-based, primary portal monitors a challenging problem.

An important component of the ALPS project is a systematic study of the dependence of scintillator energy resolution and gamma-detection sensitivity on light collection efficiency. Although the optimum energy resolution of plastic is limited by the number of light quanta emitted per unit of deposited energy, poor light collection in the current generation of portal monitors essentially wastes at least an order of magnitude in light signal. To the extent that the light-collection efficiency can be improved, say by a multiplicative factor, F, the resolution of the energy-deposition spectrum can be expected to improve by a factor of $\mathrm{F}^{1 / 2}$. The configuration of PMTs on each plastic scintillator sheet of the ALPS sensor will permit light-collection efficiencies of approximately $40 \%$, representing an order of magnitude improvement in light collection over single-tube sensors, and a factor of roughly 6 improvement over existing two-tube 
sensors. The energy-deposition resolution attending this increase in light collection is thus expected to be approximately a factor of 3.4 over single-tube sensors and a factor of 2.4 over two-tube sensors.

Another important factor limiting the precision of radionuclide-identification capability in plastic is nonuniformity of light collection as a function of position. In many commercial instruments, only one PMT is used to collect the scintillation light. Even in units with two PMTs, both tubes are mounted on the same end of the plastic slab. This further reduces the effective energy-deposition resolution because the light-collection efficiency can vary significantly (by as much as a factor of two) depending on the location of the radiation interaction. To achieve the highest resolution from a large-area plastic scintillator, compensating for variation in interaction position in the scintillator appears to be an appropriate strategy. One type of straightforward software correction familiar from applications of so-called "double-ended" scintillators (i.e., scintillators accommodating PMTs at each end of a long slab of plastic) in nuclearphysics experimentation is to calculate the geometric mean of the pulse heights registered from the two PMTs. This type of pulse-height-correction algorithm seeks to address, to first order, the attenuation of the light signal as it propagates to the two ends from a given position within the scintillator. However, the variation in light-collection efficiency with transverse distance in the scintillator-i.e., distance in the "width" dimension of the detector as opposed to the "length" dimension-is ignored in this type of correction. In the case of more complicated scintillant + PMT geometries, analogously more complicated position non-uniformity corrections, based on an appropriate functional combination of pulse heights from the ensemble of PMTs, can likewise be envisioned.

The relative timing of PMT signals in a multi-PMT geometry offers another possible strategy for addressing the non-uniformity problem, since timing information in a large scintillator may localize the interaction position with sufficient resolution to make a position-based software correction (implementing in turn prior knowledge, either empirical or computational, of the sensor's variation in light-collection efficiency with position) feasible. One of the goals of the ALPS research is to determine the light output of the plastic scintillator as a function of the location of the radiation interaction and to identify possible algorithms, operating either on the set of raw pulse heights alone or including timing information, for correcting the observed pulse-height variations with position. If timing information is shown to permit significant enhancement in the software correction of pulse height as a function of position, the feasibility of implementing timing information in the on-board circuitry of a field-deployable portal monitor must be assessed.

As previously discussed, gamma interactions in plastic scintillator are predominantly Compton scattering events. The possibility exists that a single gamma incident on a multi-layered sensor could deposit energy in both scintillator sheets, yielding a coincidence signal between the two layers that occurs with a probability dependent upon the energy of the incident gamma. Exploiting the possibility of successive interactions in a multi-layered sensor may provide an additional means of characterizing radionuclides. In addition, the simple addition of additional plastic material thickness will increase the overall gammadetection efficiency of the sensor by essentially a factor of two. With these considerations in mind, a third important goal of the ALPS project is to investigate the source-sensitivity advantages resulting from the use of a double-layered scintillator geometry.

Finally, a crucial component of the ALPS research focuses on assessing the impact of increased lightcollection and energy-deposition resolution on the capability of plastic scintillator to identify radionuclide sources and discriminate among radiological threats. Even in the extreme case of perfect energy- 
deposition resolution, i.e., the case (impossible to achieve in practice) in which the amount of energy deposited by a gamma ray in the plastic scintillant is known perfectly, information that the traditional gamma spectroscopist regards as crucial to reliable radioisotope identification - namely, the photopeakwould still be lacking in a plastic scintillator that was not inconveniently thick. The question naturally suggests itself: What is the maximum information that can be extracted from a continuum-energydeposition profile, consisting of (at best) one or more resolution-smeared Compton backscattering "peaks" (i.e., the Compton "edge" representing the greatest energy that can be transferred by a gamma to an atomic electron at 180 degrees scattering angle), with their attendant lower energy-continuum distributions? Various approaches to this question have been implemented in modern-day portal monitors. In one approach, details of the continuum shape are ignored completely, and the gross count rate above a pulse-height threshold serves as the single alarm criterion for identifying a radiological hazard. Other approaches implement so-called "energy windowing" schemes, in which the plastic scintillator spectrum is divided into appropriate regions of interest (ROIs), and various count ratios between pairs of ROIs are used to characterize the gamma source's energy distribution. The potential also exists for applying templating schemes to the identification of plastic energy deposition spectra. In this analysis scheme, a measured spectrum is compared to a library of pre-existing spectra of known gamma source and shielding characteristics. The best spectrum match (as determined by e.g. a $\chi^{2}$ minimization algorithm) yields the source identification, and the quality of the match provides confidence limits on the identification certainty.

In general, any characterization of the energy distribution in plastic scintillator (formulated, for example, as an algorithm for identifying radiological threats by triggering a portal-monitor alarm) will be susceptible to statistical count limitations inherent in the limited-time exposure typical of the standard operating procedure at a vehicle-portal-monitor installation. In the energy-windowing schemes, the number of counts registered in various ROIs will depend on several factors, including the exposure time, the source's geometrical distribution in the vehicle and shielding, and the effective energy-deposition resolution of the sensor. In the limit of very poor sensor energy resolution, the counts accumulated in each ROI window are essentially randomized, and spectral characterization of the source becomes impossible. As the energy resolution of the sensor improves, one expects that the pattern of counts registered in each ROI sharpens - that is, suffers less distortion due to statistical "sloshing" of the continuum counts from one ROI to another. If the continuum is flat as a function of energy, resolutionsmeared redistribution of counts among neighboring ROIs will tend to cancel. On the other hand, if the energy-deposition distribution varies with energy (as is the case in reality), this resolution-smeared redistribution will alter the apparent shape of the continuum. Given this type of resolution consideration in a sensor that inherently measures the characteristics of the energy deposition continuum, an important set of questions to be answered in the ALPS research includes the following: How does the gammaspectrum characterization capability (e.g., as implemented in energy-windowing algorithms) vary with scintillator resolution, especially in the case of a limited-duration exposure with inherently low counting statistics? What is the optimum strategy for spectral-distribution characterization in the absence of photopeaks? How many energy ROIs are sufficient for reliable identification of a radiological threat? And what is the effect of background variations (e.g., self-shielding of ambient background by the vehicle) and various source shielding configurations on the spectrum characterization capability in a plastic sensor?

As the first year (FY 2003) of the ALPS project draws to a close, only a subset of these research goals have been addressed. Project milestones in the first year included the following: 
- Monte Carlo modeling of the light-collection efficiency of various candidate plastic scintillator + PMT configurations was used to identify a conceptual design for a "next generation" sensor. These modeling studies were reported in Jordan et al. (2003).

- Engineering design and parts acquisition for a two-layer, multiple-PMT plastic-scintillator sensor were completed. The design permits systematic laboratory investigation of total light-collection efficiency and the variation of light-collection efficiency with position in plastic scintillator as a function of PMT number.

- Construction of the sensor and housing in a light-tight enclosure suitable for laboratory experimentation were completed. Pulse-processing electronics and data-acquisition hardware were acquired, configured, and debugged. Data-acquisition software (based on the Kmax framework) was written and implemented for convenient control of scintillator sensor measurements, with an option available for both online data processing into histograms or offline reduction of list-mode data.

- Experiments in mapping systematic gamma-response and beta-source position were conducted with a single scintillator sheet, outfitted with three configurations of PMTs: a single PMT at one end, two PMTs at one end, and one PMT at each end.

- The Monte Carlo code developed for light-simulation studies was generalized to simulate the interaction of a source of ionizing radiation with the plastic scintillator, followed by transport of the scintillation light generated by the ionizing radiation.

We report here on the experimental and modeling activities associated with the ALPS project for the second half of FY 2003. In the course of electronics setup and data-acquisition shakedown, preliminary data were obtained on a small area, $30 \times 30 \times 6 \mathrm{~cm}^{3}$ block of plastic scintillator viewed by four PMTs on the beveled corners. Those experiments, summarized in Reeder and Stephens ${ }^{(a)}$ and reproduced in this report as Appendix B, were valuable in solving data-acquisition problems in preparation for the experiments described in this report involving a much larger block of plastic scintillator. Accommodating the larger set of scintillator sheets constituting the ALPS sensor required construction of a large, lighttight box and an adjustable mechanical support framework to hold the scintillator slabs and accompanying PMTs in place. Sections II through VI of the report summarize progress on the ALPS experimental campaign, including the characteristics of the light-tight box and the data-acquisition system. The first results from the use of one or two PMTs with a single large scintillator slab are also presented. Section VII describes the generalization of the Monte Carlo simulation to fully model the interaction of a radiation source with the sensor, from the interaction of the ionizing radiation to the propagation of scintillation light to the PMTs, and modeling of the PMT signal-generation response. The report concludes with a discussion of follow-on activities anticipated for the second year of the project.

(a) PL Reeder, and DL Stephens. 2003. Response of $30 \times 30 \times 6 \mathrm{~cm}^{3}$ Scintillator to ${ }^{90} \mathrm{Sr}$ as a Function of Source Location. PNNL Internal Report; reproduced as Appendix B in the current report. 


\subsection{Experimental: Apparatus}

\subsection{Equipment}

The project apparatus is designed to allow maximum flexibility in experimental setup. The system is designed around two Bicron/Saint Gobain BC-408 plastic scintillators of dimensions $127 \times 57.15 \times$ $5.08 \mathrm{~cm}^{3}$. Each scintillator can accommodate up to six Hamamatsu R1250 5-in. (127-mm) diameter photomultiplier tubes (Hamamatsu catalog number H6527 with integral tube base). Each scintillatorPMT assembly is mounted on an elevator that allows independent vertical motion of the entire assembly over a range of approximately $25 \mathrm{~cm}$. The entire apparatus is enclosed in a light-tight box with a composite wood-foam door that allows easy access for quick source and configuration changes. All signals and high-voltage signals are input via a light-tight patch panel. Figure 2.1 displays an engineering drawing of the complete sensor in its light-tight housing. Figure 2.2 shows photographs of the sensor as configured for tests at the completion of FY 2003.

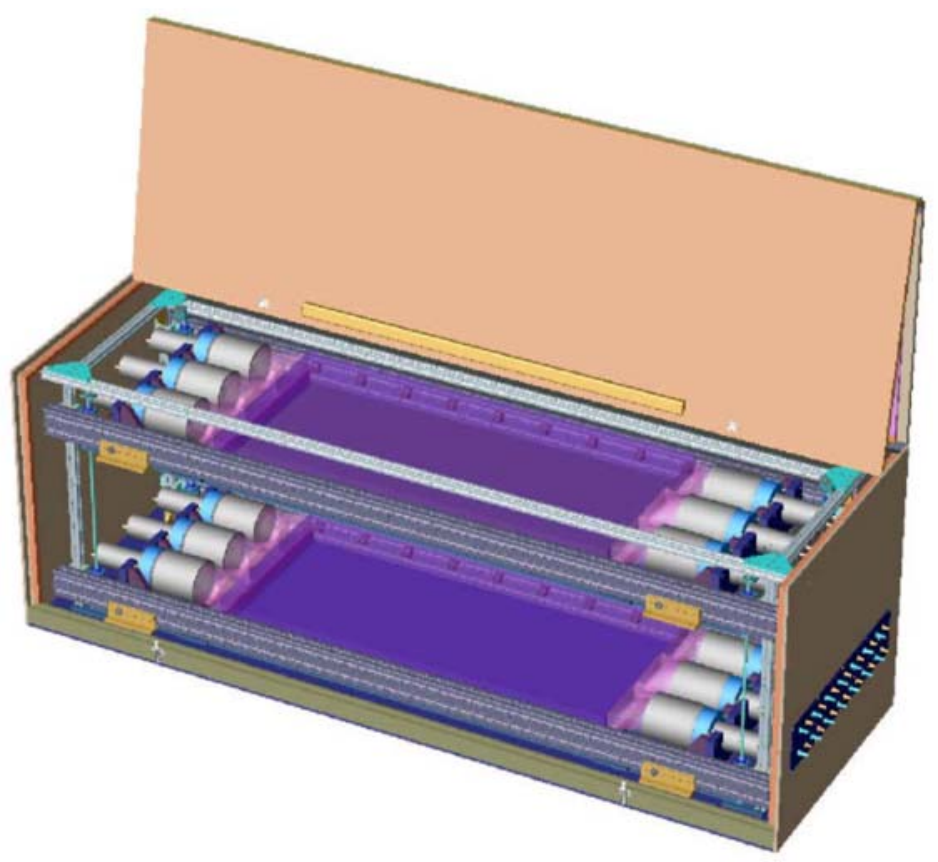

Figure 2.1. Design Drawing of the ALPS Project Apparatus Showing Two Scintillator Slabs with Three PMTs on each End of both Slabs 

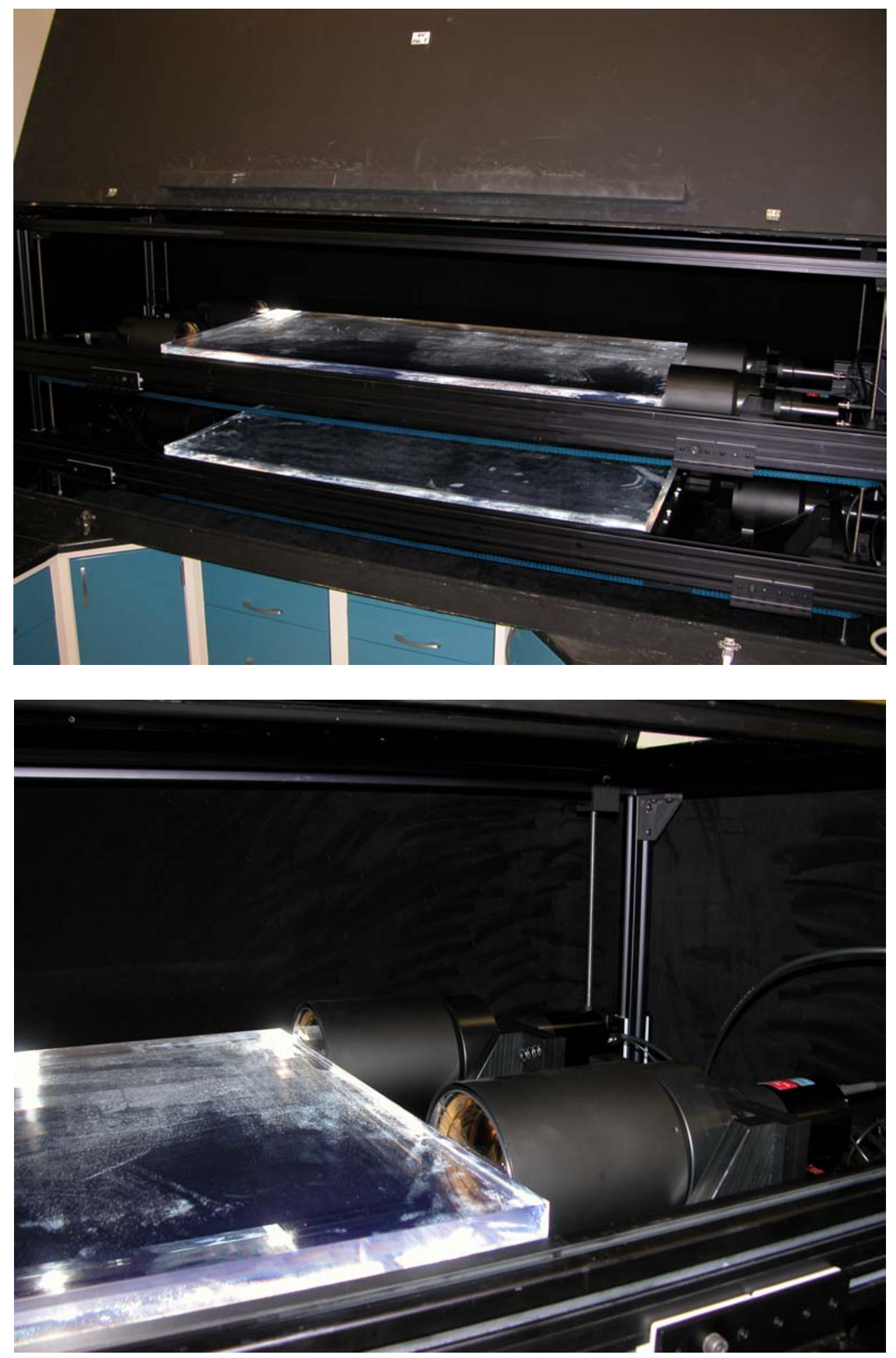

Figure 2.2. Photographs of the ALPS Scintillator Sensor as Configured for Laboratory Tests at the Conclusion of FY 2003. The upper scintillator slab is shown here outfitted with two 5-in.-diameter PMTs at one end (see Section 5). 


\subsection{Data-Acquisition System}

The data-acquisition system is composed of CAMAC and NIM data processing modules and a PC running Kmax Version 7.3 software. The linear signals from the PMTs are transferred on equal length RG-134 50 $\Omega$ cable to preserve timing. The signals go to a linear fan-out (Phillips PS748). The fan-out splits the signal and allows parallel processing of the incoming signal. The linear signals are delayed using an Ortec DB463, allowing up to $64.5 \mathrm{~ns}$ maximum delay. The delayed linear signals are then fed into a CAMAC-based Phillips 7166 Charge to Digital (QDC) converter. The other fan-out signal is sent into a Phillips PS730 discriminator operating in the ARC mode. The ARC mode uses the lower level discriminator to establish the arrival time of the pulse and the upper level discriminator to establish the acceptance threshold. In this work, the pulses greater than $60 \mathrm{mV}$ were accepted, but the timing was set when the pulse crossed the $30-\mathrm{mV}$ level. The discriminator output is then split into two signals. One signal is delayed and sent to the CAMAC based Phillips 7186 Time to Digital (TDC) converter, and the second is sent to the system master-gate logic.

The master-gate logic consists of Ortec CO4020 logic units and LeCroy 622 logic units. In the experiments conducted to date, only the Ortec units are used. In the case of the two PMT experiments, a coincident signal condition is required to produce a master gate. The gate is then passed to both the QDC and TDC. The gate width is $200 \mathrm{~ns}$ to accommodate the arrival of linear signals and is operated in the non-updating mode.

The CAMAC units are in a Jorway CAMAC mini-crate with a Jorway 73A crate controller. To accommodate the high event rates associated with large plastic scintillators, a Hytec LP1342 list processor CAMAC module is used. This module acts as a buffer by responding to look-at-me (LAM) requests from the CAMAC modules and issuing commands to read and store the data. Once the list processor has filled its memory (approximately 1-million 32-bit words), it requests a block transfer from the CAMAC crate to the computer via the crate controller. The data are then transferred over a fast-wide small computer systems interface (SCSI) connection via an Adaptec AHA-2940 SCSI controller to the Dell 340 workstation computer. The acquisition software is developed in Kmax to allow full runtime histogramming. This has reduced the offline processing of data to a minimum. Data are stored in listmode files as well as histograms created from Kmax.

The large scintillators are unshielded so they have background counting rates of about $3000 \mathrm{cps}$ from cosmic rays and natural radioactivities. The data-acquisition system has significant dead time even at this background level, so it is necessary to include a live-time measurement along with the pulse height and timing measurements. This is accomplished with a 60-cycle pulser feeding a QDC channel and a TDC channel in the same manner as signals from the PMTs. The ratio of observed counts in the pulser TDC channel to the expected number of counts from the pulser is the system live time. All the histograms shown below have been corrected for system live time and normalized to a common counting interval.

\subsection{Characteristics of Gamma Sources}

A set of standard gamma sources was used to calibrate the observed pulse height in channel number versus energy deposited. Gamma rays interacting in plastic scintillator do not ordinarily give a distinct photopeak. Instead, the pulse-height spectrum for a monoenergetic gamma gives a broad bump with a distribution to lower pulse heights. The broad bump and distribution is a result of Compton scattering of 
the gamma on an electron. The theoretical probability of Compton scattering has a peak at a gamma scattering angle of 180 degrees. This peak is greatly broadened by the resolution of plastic scintillators. However, an approximate energy calibration can be obtained by assuming that the centroid of the bump corresponds to the maximum recoil energy of the Compton scattered electron.

The sources used in this work are listed in Table 2.1.

Table 2.1. Gamma Sources, Energies, Maximum Energy of Compton Scattered Electron, and Gamma Abundances for Sources Used in this Work

\begin{tabular}{||l|c|c|c||}
\hline Source & $\begin{array}{c}\text { Energy } \\
(\mathbf{k e V})\end{array}$ & $\begin{array}{c}\text { Max. Compton Electron } \\
(\mathbf{k e V})\end{array}$ & $\begin{array}{c}\text { Abundance } \\
\mathbf{( \% )}\end{array}$ \\
\hline${ }^{109} \mathrm{Cd}$ & 88.03 & 22.56 & 3.63 \\
\hline${ }^{57} \mathrm{Co}$ & 122.06 & 39.46 & 85.60 \\
\hline & 136.47 & 47.51 & 10.68 \\
\hline${ }^{133} \mathrm{Ba}$ & 81.00 & 19.50 & 34.11 \\
\hline & 276.40 & 143.63 & 7.15 \\
\hline & 302.85 & 164.27 & 18.30 \\
\hline & 356.01 & 207.26 & 61.94 \\
\hline & 383.85 & 230.45 & 8.91 \\
\hline${ }^{137} \mathrm{Cs}$ & 661.66 & 477.34 & 85.10 \\
\hline${ }^{54} \mathrm{Mn}$ & 834.84 & 639.21 & 99.98 \\
\hline${ }^{22} \mathrm{Na}$ & 511.01 & 340.68 & 160.68 \\
\hline & 1274.54 & 1061.71 & 99.94 \\
\hline${ }^{60} \mathrm{Co}$ & 1173.23 & 963.42 & 99.86 \\
\hline & 1332.49 & 1118.10 & 99.98 \\
\hline${ }^{232} \mathrm{U}$ & 583.19 & 405.53 & 30.60 \\
\hline & 2614.51 & 2381.76 & 35.86 \\
\hline
\end{tabular}




\subsection{Results for Single PMT}

The first experiments involved the use of a single PMT located in the middle of the 57.15 -cm side (i.e. the short side). As many commercial portal monitors use this configuration, we used this same configuration to establish a baseline against which we can compare alternative configurations using more PMTs.

\subsection{Gamma Sources}

The gamma sources were mounted outside the black box at a total distance of $66 \mathrm{~cm}$ above the center of the plastic scintillator. The high voltage of the PMT was adjusted so that the highest channel in the pulseheight spectrum was about $2 \mathrm{MeV}$. Examples of pulse-height spectra from ${ }^{133} \mathrm{Ba},{ }^{137} \mathrm{Cs}$, and ${ }^{54} \mathrm{Mn}$ sources are shown in Figure 3.1. Note that each source has a distinctive distribution, but that the Compton 180 -degree scatter peaks are not very prominent. Other sources measured included ${ }^{60} \mathrm{Co},{ }^{232} \mathrm{U},{ }^{22} \mathrm{Na},{ }^{57} \mathrm{Co}$, and ${ }^{109} \mathrm{Cd}$.

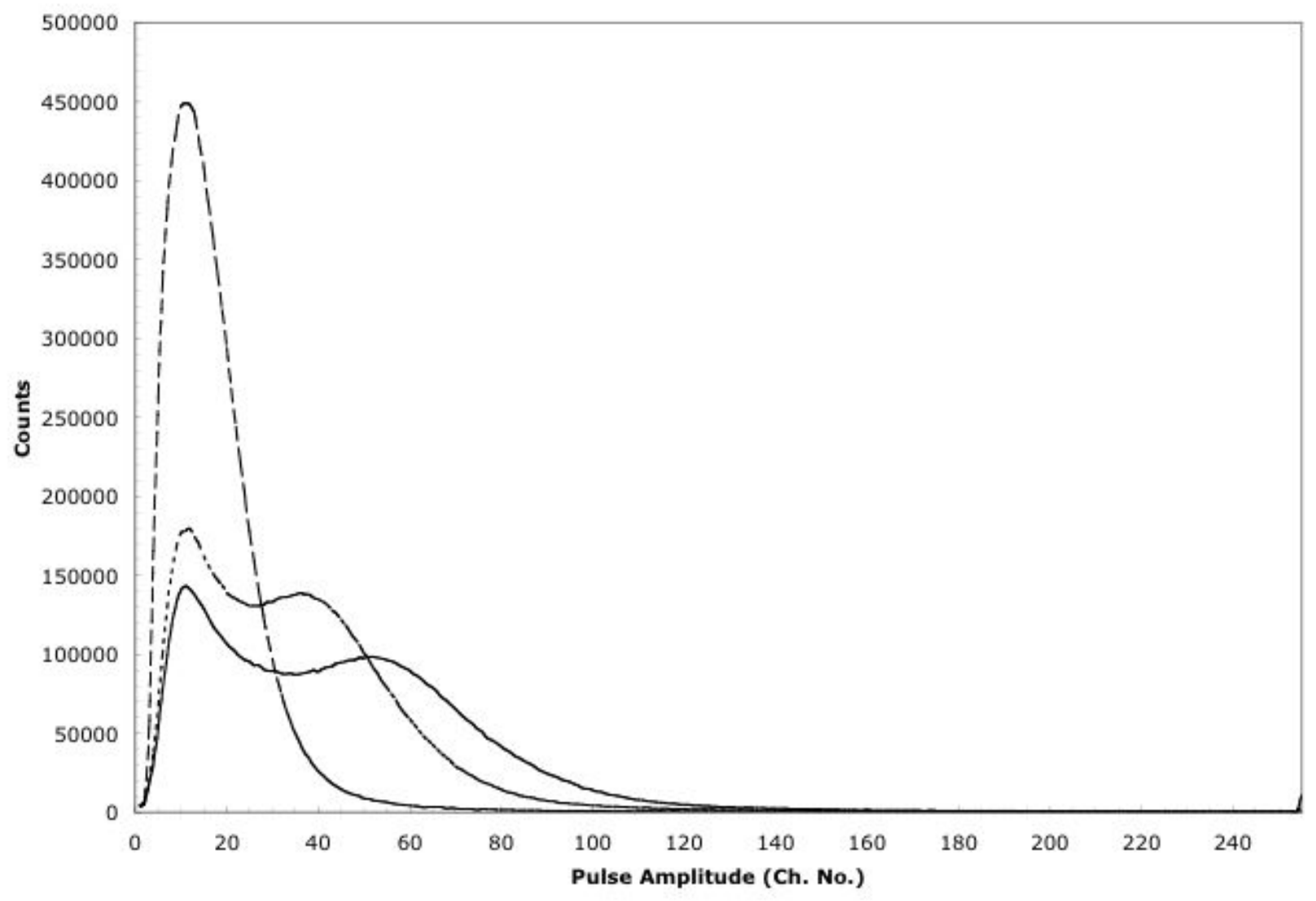

Figure 3.1. Gamma Pulse Height Spectra for Single PMT Configuration. Solid curve is ${ }^{54} \mathrm{Mn}$. Short dash curve is ${ }^{137} \mathrm{Cs}$. Long dash curve is ${ }^{133} \mathrm{Ba}$. 


\subsection{Beta Source}

A laboratory determination of the scintillator response as a function of location can be accomplished by moving a collimated radiation source to various locations and recording the pulse-height response at each location. Gamma sources are difficult to collimate to a specific location, so we used a ${ }^{90} \mathrm{Sr} /{ }^{90} \mathrm{Y}$ beta source restricted to a 1-cm-diameter region by an aluminum collimator. By mapping the pulse-height response as a function of the location of the collimated source, we can generate an empirical response matrix that includes corrections for light loss by absorption, changes in light-collection efficiency, and multiple reflections.

However, in a field instrument, there must be some technique that allows determination of the location for each interaction based solely on the light output from that event. Event location in a large plastic scintillator can be accomplished if multiple PMTs are used to collect the light. At least two different approaches can be employed. One is to look at the ratio of pulse amplitudes in different PMTs, and the other is to look at differences in signal arrival times in different PMTs. In our previous work, ${ }^{(a)}$ we noted that ratios of pulse amplitudes in rectangular blocks of scintillator can be distorted by the fact that multiple reflections contribute a large fraction of the pulse amplitude. In this work, we are investigating whether arrival-time information can be more reliable because it is less sensitive to multiple reflections of the scintillation light.

The collimated beta source was measured at 12 locations along the mid-point axis. Examples of the beta spectra at three locations are shown in Figure 3.2. Note that there appear to be two exponential components in the pulse-height distributions as expected for the ${ }^{90} \mathrm{Sr} /{ }^{90} \mathrm{Y}$ beta spectra. As the source is moved farther from the PMT, more events appear in the low-energy region, and fewer events appear in the high-energy region. It is clear that light attenuation and solid angle for light-collection effects cause a significant decrease in the observed pulse heights when the radiation interaction occurs farther from the PMT.

To illustrate the dependence of the pulse amplitude as a function of the location of the event, we calculated the mean value of the beta distributions at each location. The mean values were calculated after correcting the gross beta spectra for background. These mean values are plotted as a function of distance from the PMT in Figure 3.3. Note that there is more than a factor of two difference in mean pulse height when the source is moved from one end of the scintillator to the other. Unless some correction is made for the location of the event, the spectrum due to uniform irradiation of the scintillator will have poor resolution.

(a) PL Reeder, and DL Stephens. 2003. Response of $30 \times 30 \times 6 \mathrm{~cm}^{3}$ Scintillator to ${ }^{90} \mathrm{Sr}$ as a Function of Source Location. PNNL Internal Report; reproduced as Appendix B in the current report. 


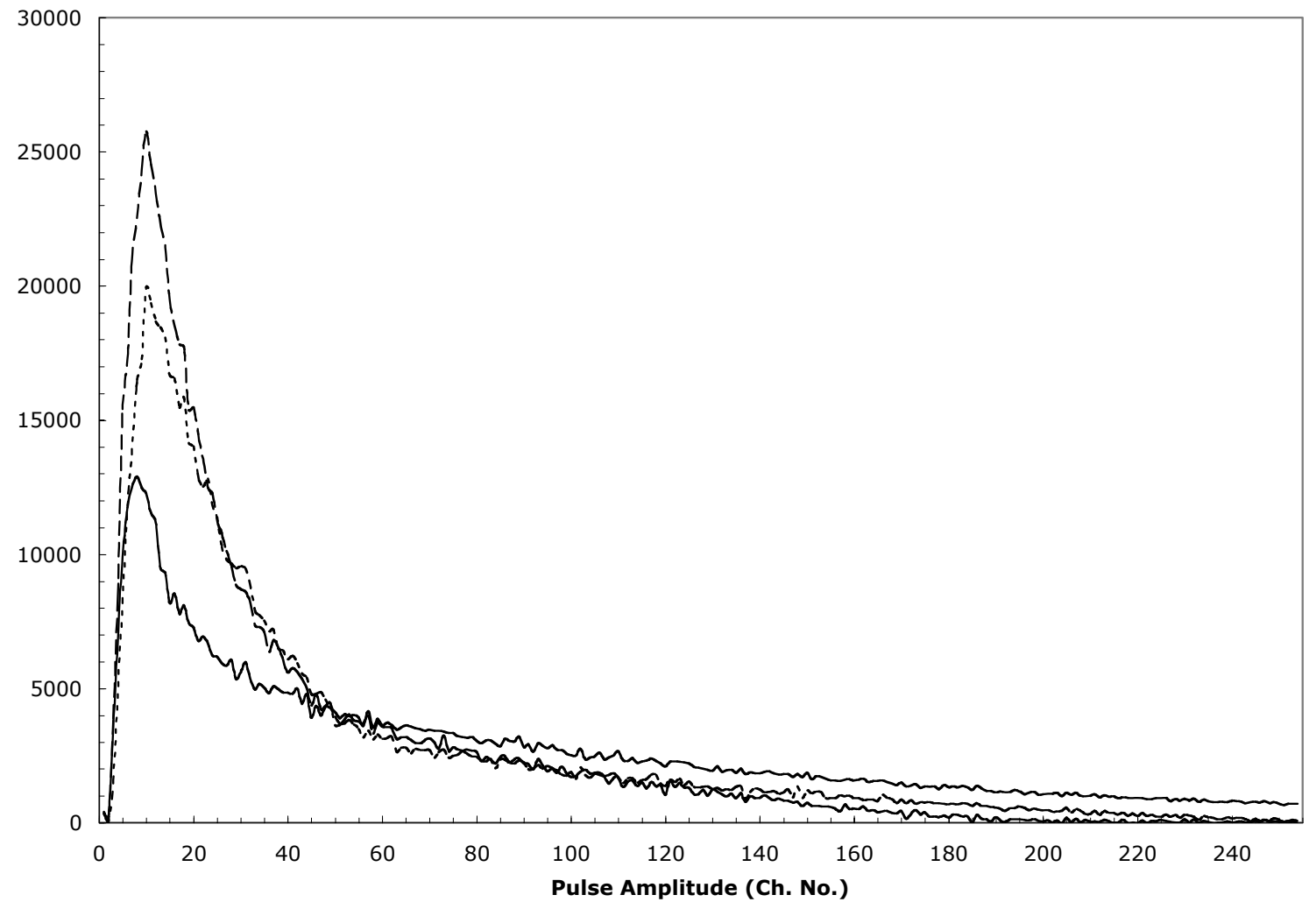

Figure 3.2. Beta Pulse Height Spectra from a Single PMT at Three Locations Along Mid-Point Axis. Solid curve is source at $5.08 \mathrm{~cm}$ from PMT. Short dash curve is source at $\mathbf{5 0 . 8}$ $\mathrm{cm}$ from PMT. Long dash curve is source at $121.9 \mathrm{~cm}$ from PMT.

Also shown in Figure 3.3 are the mean values of the beta distributions for locations on an axis parallel to the mid-point axis but offset by $17.8 \mathrm{~cm}$. If the interaction occurs at the far end of the scintillator, there is not much difference in the pulse height on or off the mid-point axis. However, if the source is very close to the end with the PMT, the light that gets into the PMT from the off-axis location is the result of multiple reflections with consequent light losses. For locations off the mid-point axis, a measurement of the mean value of the pulse-height distribution could correspond to locations at opposite ends of the scintillator. The obvious conclusion from these data is that a single PMT is not capable of providing a uniform response to radiation interacting at various locations in a large scintillator. 


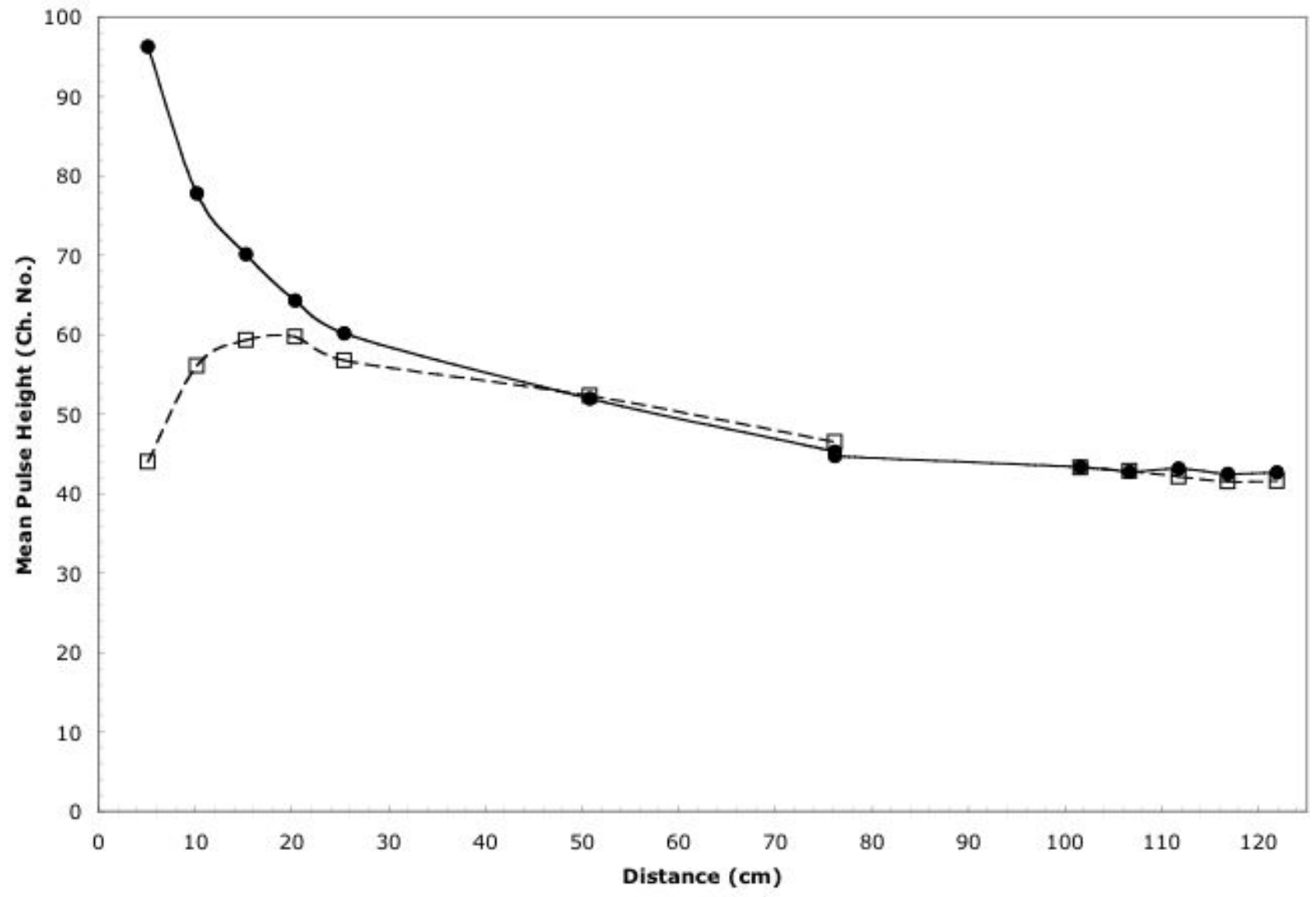

Figure 3.3. Mean Channel Number of Pulse Height Distributions for Beta Source at Various Distances from the Single PMT. Solid curve shows locations on mid-point axis of scintillator. Dashed curve shows locations along an axis $17.8 \mathrm{~cm}$ from mid-point axis. 


\subsection{Results for Two PMTs-Opposite Ends}

The second configuration consisted of the same large scintillator viewed by single PMTs located at the middle of each of the two $57.15-\mathrm{cm}$ sides. The data-acquisition system accepted only those events where coincident pulses were observed in the two PMTs. The high voltages on the PMTs were adjusted so that identical gains were obtained from each PMT. This was achieved by matching the pulse-height spectra from the individual PMTs when only background radiation was present.

\subsection{Gamma Sources}

The gamma sources were mounted on top of the black box at a distance of 35.6-cm above the center of the plastic scintillator. Again, the highest channel in the pulse-height spectrum was about $2 \mathrm{MeV}$. Examples of pulse-height spectra for the sum of the pulses from each PMT from ${ }^{133} \mathrm{Ba},{ }^{137} \mathrm{Cs}$, and ${ }^{54} \mathrm{Mn}$ sources are shown in Figure 4.1. Note that the Compton 180-degree scatter peaks are much better defined, especially for the ${ }^{137} \mathrm{Cs}$ and ${ }^{54} \mathrm{Mn}$ sources. Other sources measured included ${ }^{22} \mathrm{Na},{ }^{109} \mathrm{Cd}$, and ${ }^{232} \mathrm{U}$.

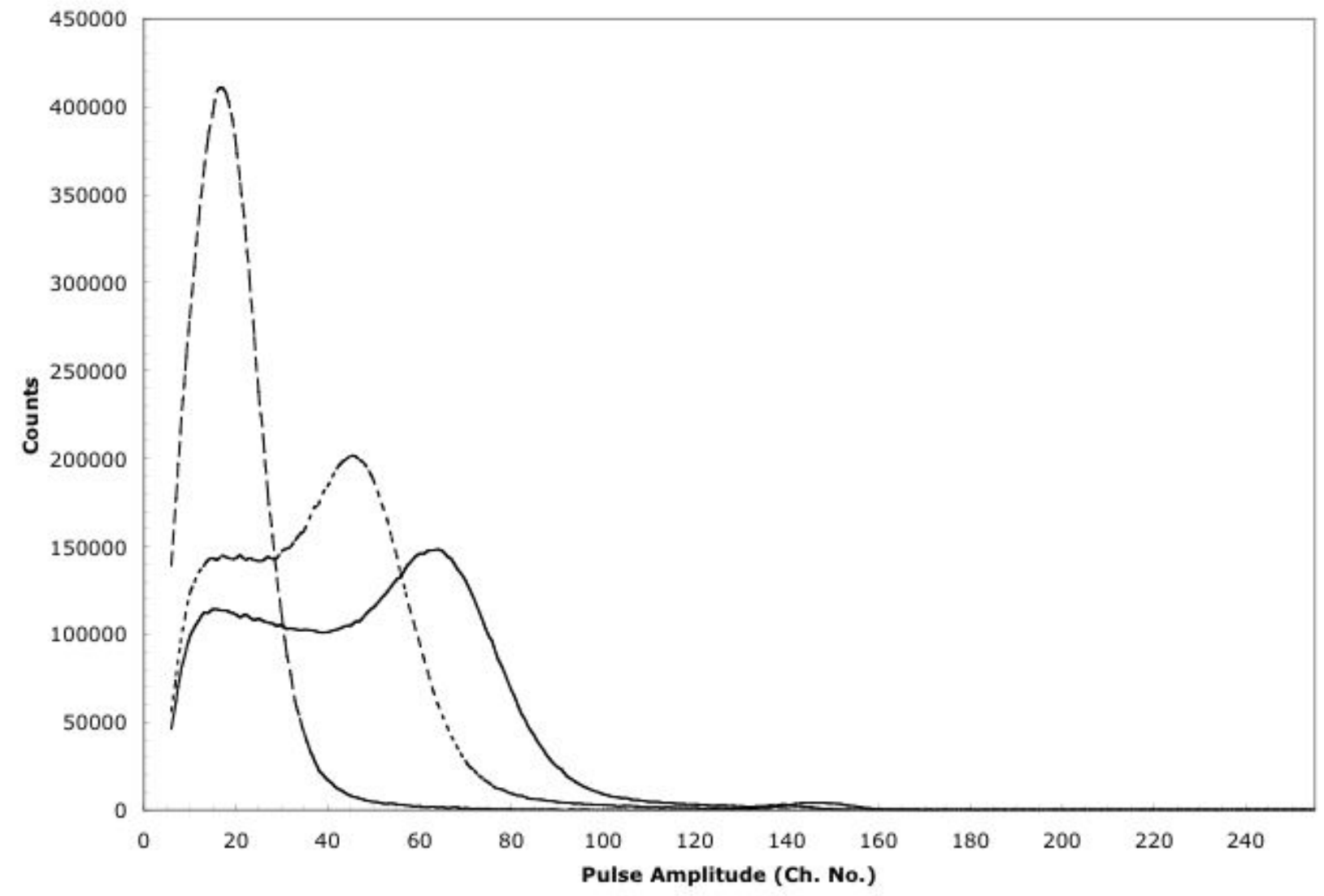

Figure 4.1. Gamma Pulse Height Spectra for Sum of Pulses from each PMT for Configuration with a PMT on Opposite Ends. Solid curve is ${ }^{54} \mathrm{Mn}$. Short dash curve is ${ }^{137} \mathrm{Cs}$. Long dash curve is ${ }^{133} \mathrm{Ba}$. 


\subsection{Beta Source}

The collimated ${ }^{90} \mathrm{Sr} /{ }^{90} \mathrm{Y}$ beta source was measured at several locations along the mid-point axis and along an axis parallel to the mid-point axis but offset by $17.8 \mathrm{~cm}$. Mean values of the net beta-pulse-height spectra were calculated for the individual PMTs and for the sum of the two PMTs. The data taken along the mid-point axis are plotted in Figure 4.2. Note that the mean values of the individual PMTs decrease with distance in the same manner as for the single PMT described above. The mean values of the sum spectra are approximately equal to the average of the mean values of the individual PMT spectra. Because of the large enhancement in pulse height close to one PMT, the mean value of the sum spectra is about a factor of two greater near the PMTs than it is in the middle of the scintillator. Even by adding pulses from both ends of the scintillator, one does not get uniform light-collection efficiency.

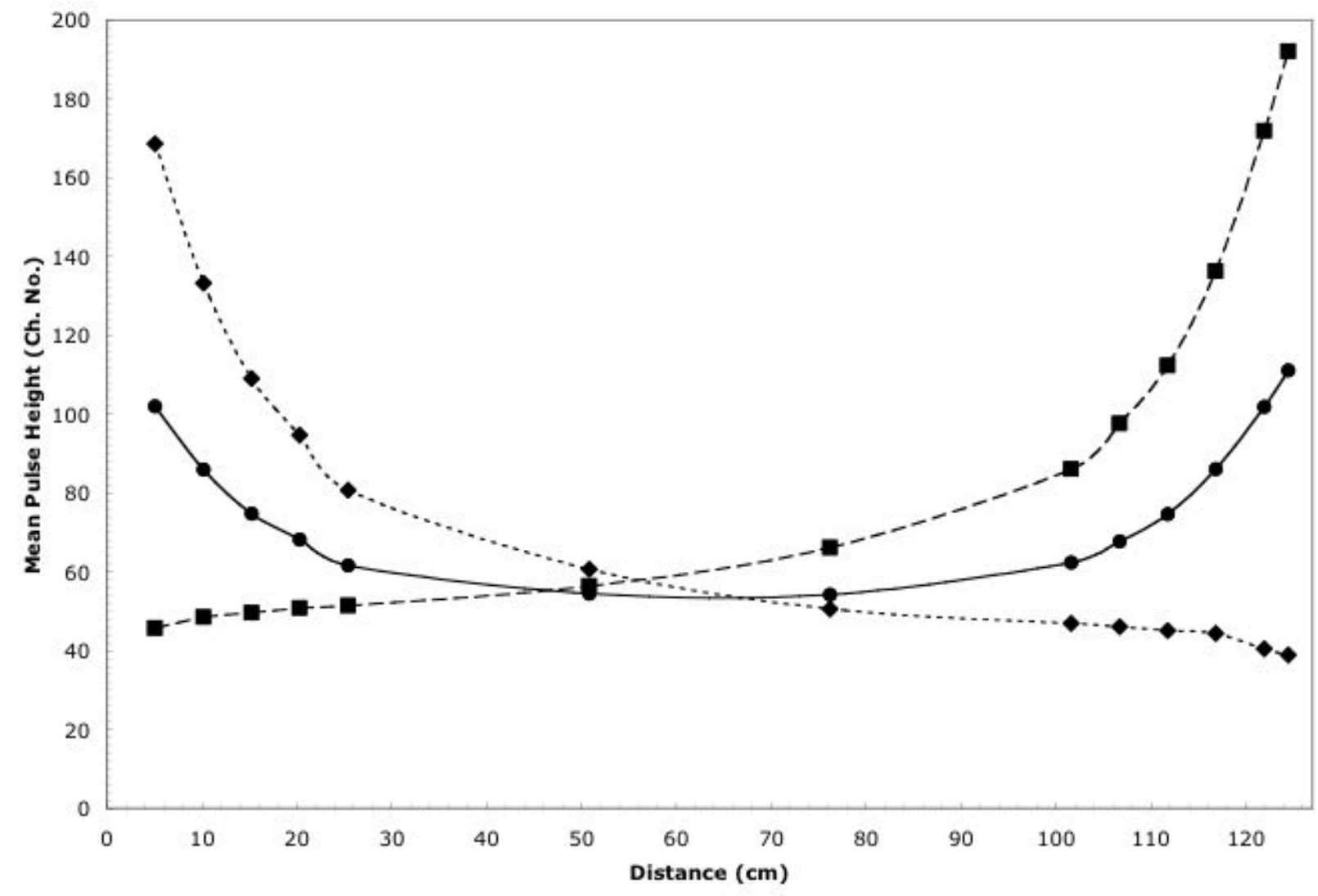

Figure 4.2. Mean Channel Number of Pulse Height Distributions for Beta Source at Various Distances Along the Mid-Point Axis Between Coincident PMTs at Opposite Ends of the Scintillator. Solid curve (circles) is mean channel of the sum spectra. Short-dashed curve (diamonds) is the mean channel of the PMT at the origin end. Long-dashed curve (squares) is for the PMT at the far end.

The data taken off the mid-point axis are plotted in Figure 4.3. The mean channel for a single PMT increases and decreases in a similar manner as shown above for the off-axis data. However, the coincidence requirement does seem to alter the response at the distant end. The result for the mean channel of the sum is a rather constant value as a function of distance along the off mid-point axis. 


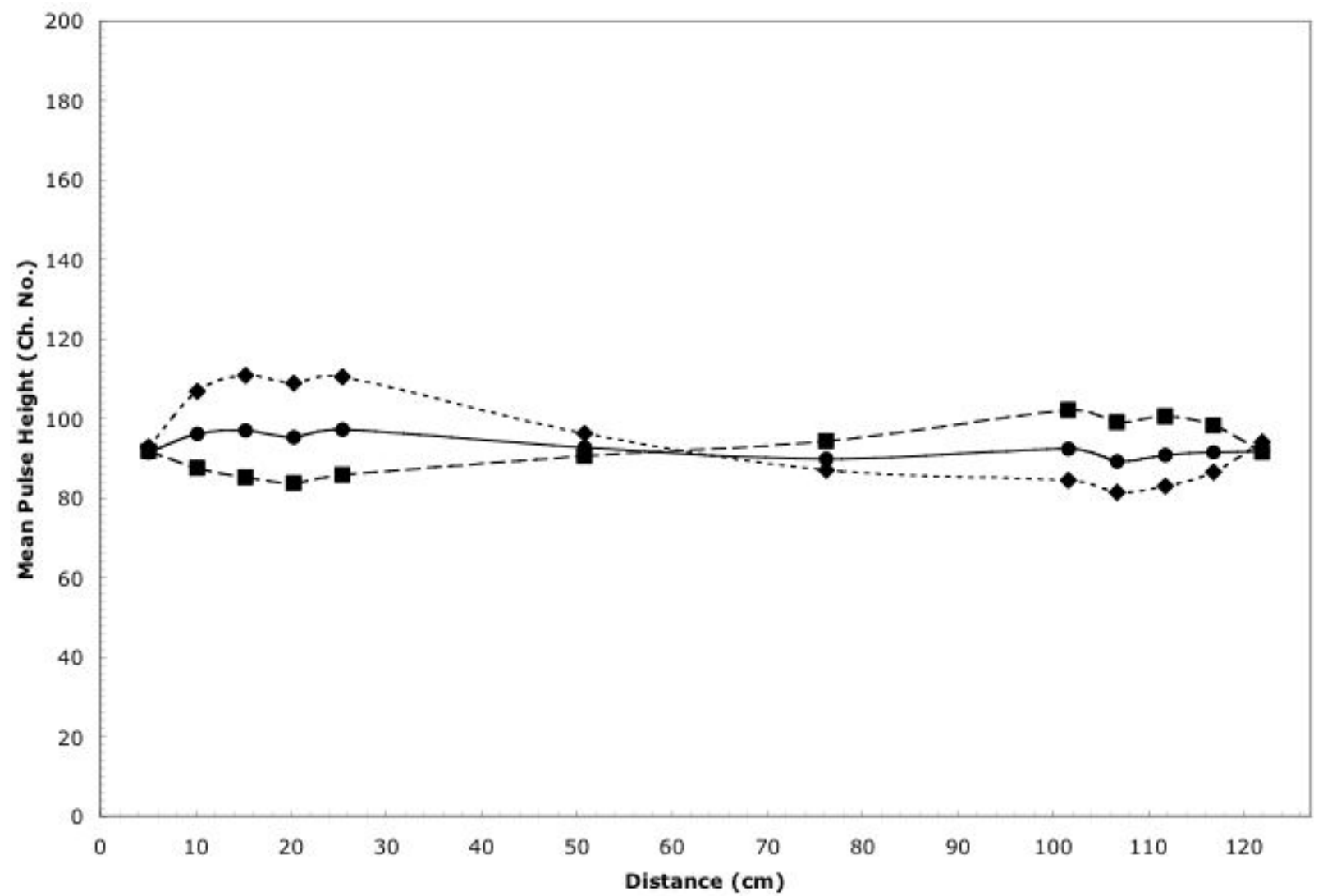

Figure 4.3. Mean Channel Number of Pulse Height Distributions for Beta Source at Various

Distances Along the Axis Parallel to the Mid-Point Axis But $17.8 \mathrm{~cm}$ away from it. The configuration is coincident PMTs at opposite ends of the scintillator. Solid curve (circles) is mean channel of the sum spectra. Short-dashed curve (diamonds) is the mean channel of the PMT at the origin end. Long-dashed curve (squares) is for the PMT at the far end. 


\subsection{Results for Two PMTs - Same Ends}

The third configuration had two PMTs mounted on the same 57.15-cm side. These two PMTs were located at $17.8 \mathrm{~cm}$ on either side of the mid-axis. Only coincident events were accepted by the dataacquisition system. Gains of the two PMTs were equalized by adjusting the high voltages.

\subsection{Gamma Sources}

The gamma sources were placed on top of the light-tight box at a distance of $35.6 \mathrm{~cm}$ from the center of the plastic scintillator. Again, full scale on the pulse height spectrum corresponded to about $2 \mathrm{MeV}$. Examples of pulse-height spectra from ${ }^{133} \mathrm{Ba},{ }^{137} \mathrm{Cs}$, and ${ }^{54} \mathrm{Mn}$ sources are shown in Figure 5.1. Note that only the ${ }^{54} \mathrm{Mn}$ source shows a distinct 180 -degree Compton scatter peak and that the plateau to the lowenergy side of the peak is enhanced relative to the second configuration discussed above. Other sources measured included ${ }^{22} \mathrm{Na}$ and ${ }^{232} \mathrm{U}$.

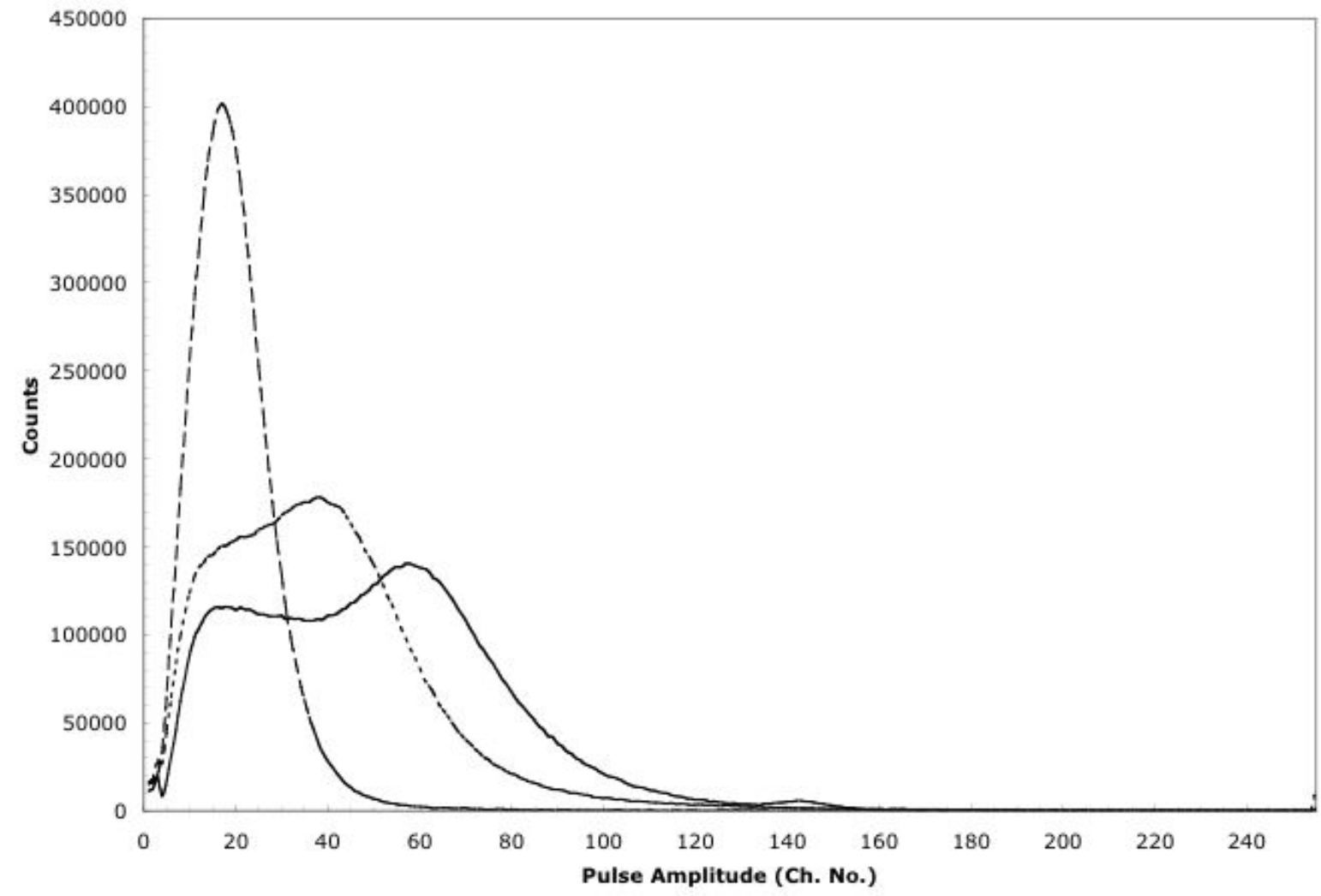

Figure 5.1. Gamma Pulse Height Spectra for Configuration with two PMTs on same End. Solid curve is ${ }^{54} \mathrm{Mn}$. Short dash curve is ${ }^{137} \mathrm{Cs}$. Long dash curve is ${ }^{133} \mathrm{Ba}$. 


\subsection{Beta Source}

The collimated beta source was measured as a function of distance from one end of the scintillator along two axes. One axis was the midpoint axis, and the other was a parallel axis away from the center line but directly in line with one of the PMTs. The results for the mean channel of the pulse-height distribution as a function of location along the midpoint axis are shown in Figure 5.2. When the source is close to the end with the PMTs, the scintillation light reaches the PMTs only after several reflections. Consequently, the pulse-height distribution is shifted to lower energies. As the source is moved further down the axis away from the edge with the PMTs, it becomes easier for the light to reach the PMTs, and the pulseheight distribution moves to higher energies. Finally, as the source is moved even further away, light losses occur due to self-absorption and scattering, so the pulse-height distribution shifts to lower energies. Both PMTs are equidistant from the midpoint axis, and so they have nearly identical responses. Likewise, the distribution of pulse heights for the sum of the two pulses has a similar response.

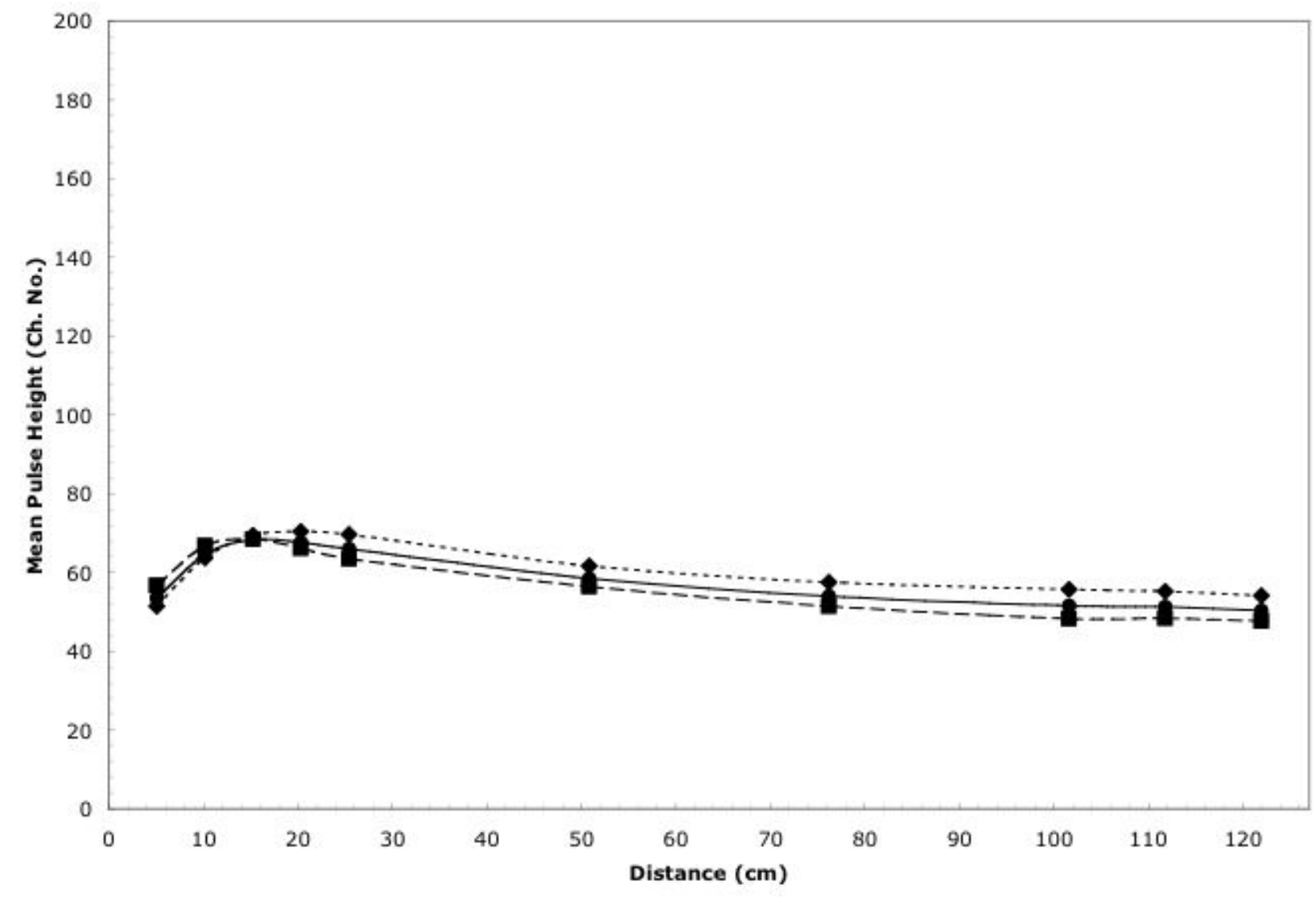

Figure 5.2. Mean Channel Number of Pulse Height Distributions Along Midpoint Axis for Configuration with two PMTs on the same End of the Plastic Scintillator. Solid curve (circles) is mean channel of the sum spectra. Short-dashed curve (diamonds) is the mean channel of the PMT at $\mathbf{- 1 7 . 8} \mathrm{cm}$ from midpoint axis. Long-dashed curve (squares) is for the PMT at $+\mathbf{1 7 . 8} \mathrm{cm}$ from midpoint axis.

The mean channel numbers for the pulse-height distributions along the axis in line with the PMT at $-17.8 \mathrm{~cm}$ from the midpoint axis are shown in Figure 5.3. For the PMT directly in line with the source, 
the mean channel numbers are large when the source is close to the PMT and fall off rapidly as the source is moved farther away. This behavior is consistent with the other measurements described above when the source is moved in line with the PMT. The other PMT has difficulty seeing the pulse when it is close to the side with the PMTs, so the mean channel numbers increase as the source moves farther away and then decrease as the source gets even farther away. The mean channel number for the sum of the two PMTs is approximately the average of the mean channels of the individual PMTs. This configuration illustrates once more the large enhancement in pulse height when the source is directly in front of a PMT.

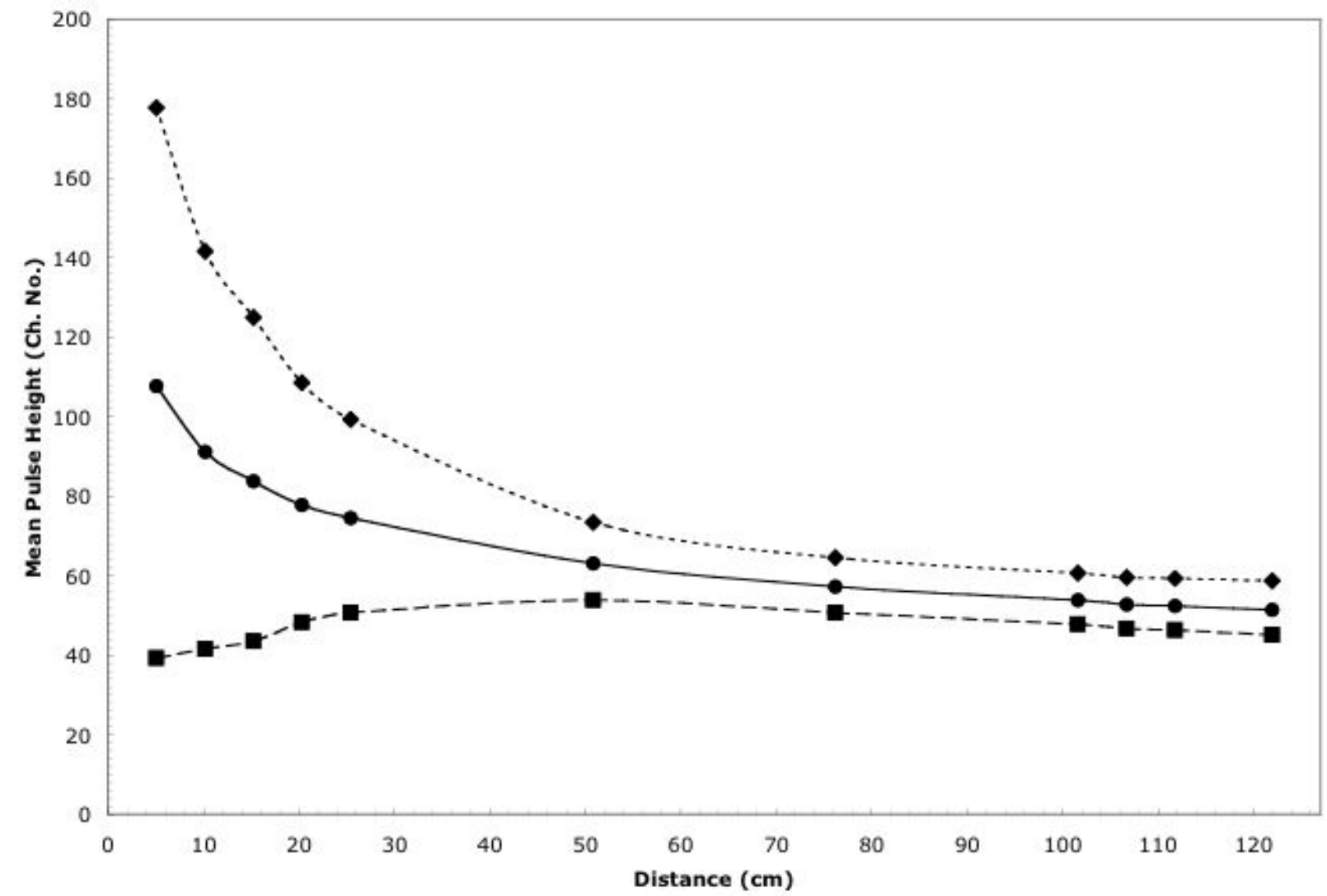

Figure 5.3. Mean Channel Number of Pulse Height Distributions Along Axis Parallel to Midpoint Axis at $\mathbf{- 1 7 . 8} \mathbf{~ c m}$ for Configuration with two PMTs on the same End of the Plastic Scintillator. Solid curve (circles) is mean channel of the sum spectra. Short-dashed

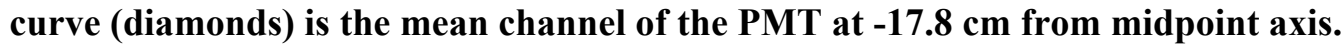
Long-dashed curve (squares) is for the PMT at $+17.8 \mathrm{~cm}$ from midpoint axis. 


\subsection{Time Difference Analysis}

Energy resolution in plastic scintillators is a function of light-collection efficiency. For a given amount of energy deposited by an ionizing particle, the number of photons created in the scintillator is constant within some statistical variation. The loss of photons before being counted degrades the resolution. Three possible effects contribute to this loss. One is the self-attenuation of photons in the scintillator media, the second is the reflective losses at interfaces, and the third is the solid angle of acceptance of the PMT. This leads to a non-uniformity of total photons detected for large scintillators as a function of event position. The method selected for correcting these losses is timing of photons in the scintillator to determine the position of the original ionizing event. This in turn allows corrections to the pulse height for the known non-uniformity of the light-collection efficiency at each of the photon detectors.

In the case of a large sheet of scintillator where the aspect ratio of the thickness $(z)$ to the width $(y)$ and length $(x)$ is small, the position is defined in the $x-y$ plane. Photon time of flight is used to determine the position of the ionization event. By observing the relative arrival times of photons at multiple points on the sheet, the position can be uniquely determined. For the plastic scintillator selected for this investigation, the velocity of light in the media is $189.742 \mathrm{~mm} / \mathrm{ns}$. Therefore, the largest flight time along the diagonal of the sheet is approximately $7.3 \mathrm{~ns}$. To resolve points $2.5 \mathrm{~cm}$ distant from each other, a time resolution of approximately $130 \mathrm{ps}$ is required.

To correct for light lost to the various processes above, the real spatial variation of pulse height for each PMT must be measured. This is accomplished with a known, mono-energetic, well-collimated source placed at points spanning the entire scintillator surface. The measured response is converted into correction factors that can then be applied to data for each event to correct for light losses and to ultimately increase energy resolution.

\subsection{Calibration of Location Versus Time Difference}

Unique position determination is required for the outlined method of resolution enhancement. This is accomplished by measuring the relative arrival times at each PMT of photons from an ionization event. The TDC is operated in a common start mode where the timer is started by the common master gate. Each timer is stopped individually by the discriminator input signal of each PMT. The total flight time is not known, but the difference between the times for each PMT can be correlated with the location of the event. The techniques used here assume that the leading edge of the PMT signal is generated by the photons traveling in the line-of-sight from the point of production to the PMT. Any photons traveling along a reflected path are in the distributed tail of the signal.

The time-distribution histograms for the collimated beta source at two locations separated by $2.54 \mathrm{~cm}$ are shown in Figure 6.1. Each time channel in Figure 6.1 is 25 ps, corresponding to the inherent best resolution of the Phillips 7186 TDC. The instrumental time resolution was determined to be about $150 \mathrm{ps}$ full width at half maximum (FWHM) by use of a pulser. The FWHM of the time distributions shown in Figure 6.1 is about $1 \mathrm{~ns}$, but the centroids of the distributions clearly distinguish the locations. 


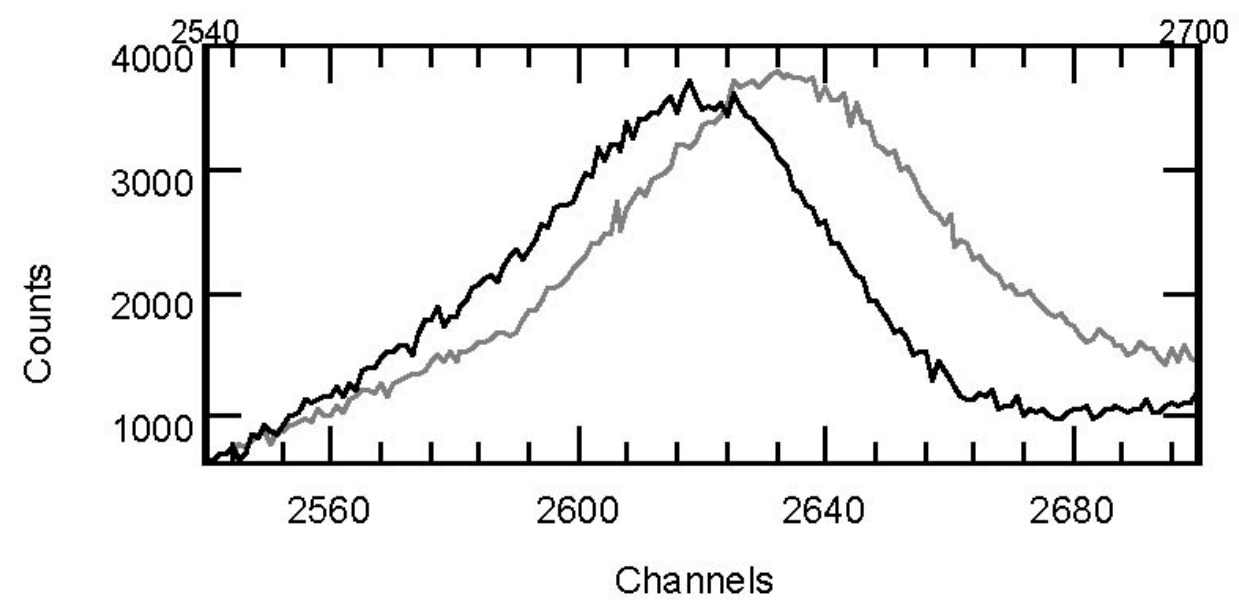

Figure 6.1. Time-Distribution Histograms of Two Beta Spectra Collected at 2.54cm Separation on Scintillator

The calculation of position from relative arrival times for multiple PMTs results in a complex set of simultaneous quadratic equations. The analytic solutions are not particularly useful for real-time corrections to the pulse height due to their complexity and multiple roots. For this reason, a look-up table for relative times will be used. This table can be calculated using Monte Carlo methods or determined empirically for use in the data-acquisition software. The table in its full form for six PMTs will be spaced on a $2.5-\mathrm{cm}$ grid due to the limit of experimental time resolution.

The double PMT experiments conducted to date use a simple determination of position along the centrallong axis $(x)$ for corrections. The beta source was placed at various positions along the central and adjacent axis as well as at selected additional points along the $y$ axis. The mean channel number of the net beta time-difference histograms was calculated for each location. The calibration of time-difference to position $\mathrm{x}$ is shown in Figure 6.2 for positions along the central axis.

\subsection{Pulse-Height Corrections Based on Location}

Investigations of a correction to pulse height derived from TDC timing-based location information are underway in the configuration with one PMT at each end of the scintillator. The algorithm for the correction is as follows: First the location of the event is determined using the time difference for each event and the calibration curve shown in Figure 6.2. The pulse height of each PMT is corrected for internal attenuation by applying the following formula:

$$
\mathrm{A}_{0}=\mathrm{A}_{i}\left[E X P\left[-\alpha \cdot l_{i}\right]\right]^{-1}
$$

where $\mathrm{A}_{0}=$ corrected pulse height

$\mathrm{A}_{i}=$ uncorrected pulse height

$\alpha=$ reciprocal of the mean free path of a photon in the media

$l_{i}=$ path length traveled by the photon. 
Two PMT position by using timing on PMT 2-5 (center) line

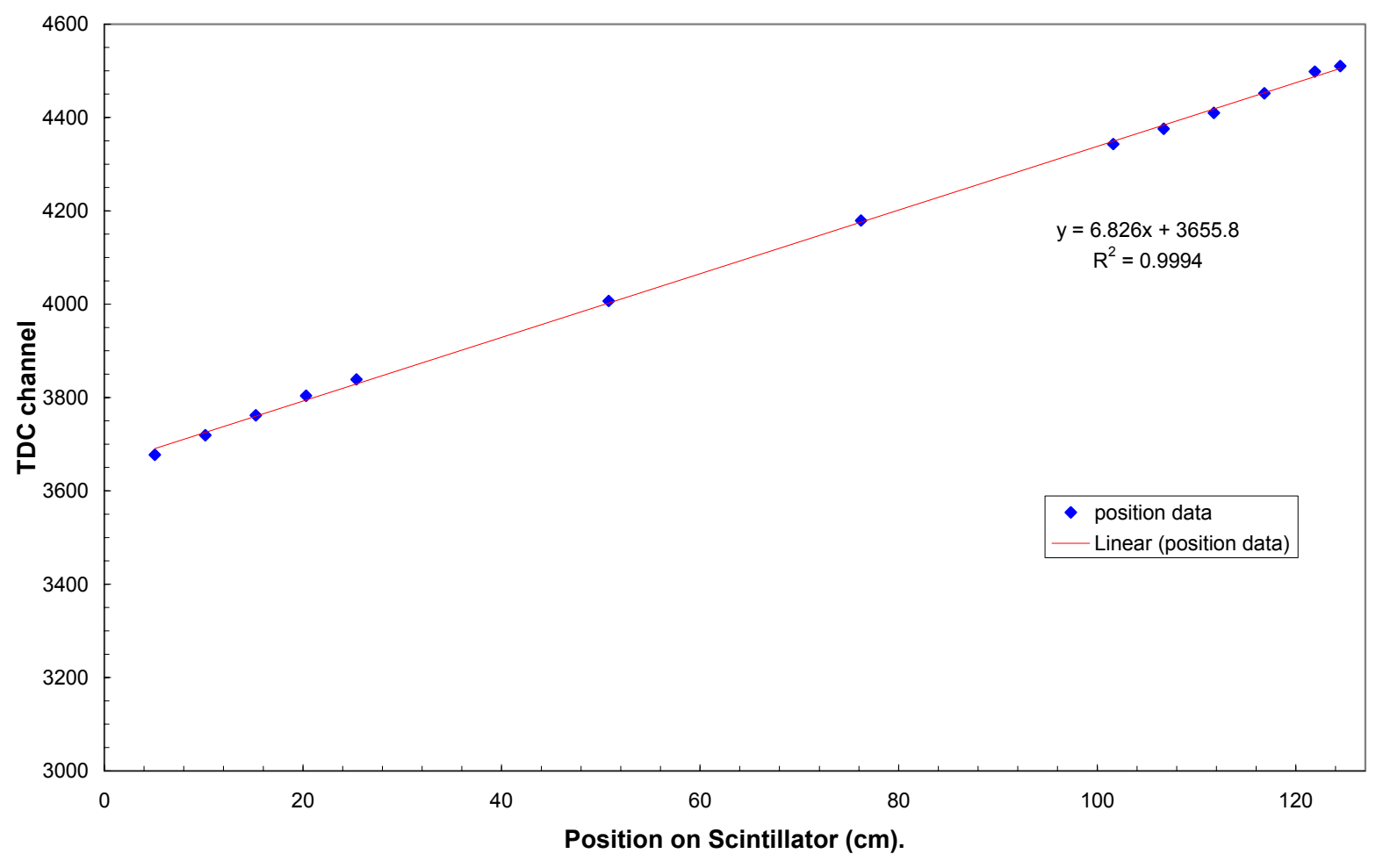

Figure 6.2. Calibration Curve for Time Difference Versus Position for the Configuration with one PMT on each End of the Scintillator

The corrected pulse heights for each PMT are then summed and divided by two to obtain the corrected sum pulse height. The expected result of this correction method is minimized variation in pulse-height spectra as a function of position on the scintillator. The correction algorithm is not yet robust against overflow of the ADC for events occurring close to one PMT, and definitive conclusions as to its effectiveness are not yet possible. Work on the correction is ongoing at the conclusion of FY 2003. This method does not attempt to make correction for geometric losses as they require both $\mathrm{x}$ and $\mathrm{y}$ position determinations. This will be explored in the planned 4 and 6 PMT configurations. 


\subsection{Simulation}

The initial stage of numerical modeling in support of the ALPS sensor design focused on the lightcollection properties of various configurations of large-area plastic scintillant slabs and accompanying PMT readout. These studies have been described in Jordan et al. (2003). Optical transport in plastic scintillator was modeled using a Monte Carlo code based on the Geant4 radiation transport simulation framework (Agostinelli 2003). The general strategy implemented in these simulations involves populating a specified scintillator slab with uniformly distributed, isotropically emitting points of light. Individual light rays are traced through the apparatus and subjected to various optical interactions, including reflection and refraction at interfaces between the plastic scintillant and the surrounding medium (e.g., air or a reflective wrapper or coating), and attenuation in the plastic. Rays traced to the detection surface of one of the model's PMTs are registered as "hits" or detected photons, and tracking of the ray is terminated. The light-collection efficiency, determined either as a function of position within the scintillator or averaged over all positions, is then easily calculated as the ratio of PMT hits to the number of light rays generated. In this early stage of simulations, no account was taken of the ionizingradiation interaction that produces the light.

Following on from these light-collection simulations, Monte Carlo modeling of the ALPS sensor has been extended to incorporate gamma and beta interactions with the plastic scintillant. Energy deposited in the scintillant by the incident ionizing radiation serves in turn as the source of optical photons, which are then tracked through the apparatus to determine the amount of light reaching the PMTs. In each primary radiation event, the total number of optical photons reaching each PMT is recorded, and this number serves as input for a simple numerical model of the multiplication effect due to the various amplification stages of the tube. The output of the PMT model is then scaled by an empirical factor in order to match as closely as possible the pulse-height spectra in the experimental data; this factor accounts for stages of the electronic pulse-processing chain in the experiment that are omitted in the simulation, including external amplification of the PMT pulses and digitization of the analog PMT pulse in the ADC. This model thus attempts to account for several effects that contribute to the energy resolution of the plastic scintillator spectrum, including 1) the statistical distribution of optical photons generated from a given amount of energy deposition, 2) the statistical distribution of optical photons reaching each PMT from a given site of photon generation within the scintillator, and 3) the distribution of electrons at each stage of PMT multiplication arising from photoelectrons produced at the PMT photocathode by the incident optical photons.

The conceptually straightforward computational scheme described above, which is intended to provide a relatively complete model for the combination of ionizing-radiation interactions in the scintillator and optical-photon propagation to the PMT's, in practice requires an inordinate amount of central processing unit (CPU) time for simulations of a reasonable number of incident radiation quanta. The reason for this is that the scintillation efficiency of a typical plastic scintillant, roughly 10,000 photons per MeV (Clark 1974), is large enough that even a relatively small number of primary radiation quanta can deposit sufficient energy in the scintillant to require subsequent tracking of an inconveniently large number of secondary optical photons. A substantial improvement in computational overhead can be achieved by splitting the scintillator modeling task into two parts, the simulation of light-collection efficiency and the simulation of ionizing radiation with light-collection properties of the scintillator described by a (positiondependent) lookup-table obtained from the light-collection modeling. The savings in CPU time afforded by this method arises from the fact that light-collection efficiencies are determined once at each position 
within the scintillator in the first stage of modeling, thus eliminating the need for (CPU intensive) optical photon tracking in the second stage of modeling.

As in the light-collection modeling described in Jordan et al. (2003), the Center for European Nuclear Research (CERN) radiation transport modeling package Geant 4 a provided the framework for the full simulation of ionizing-radiation interaction and optical-photon response described in this report.

Appendix A, "Details of the ALPS Simulation," supplies specifics of the simulation geometry specification, source terms, physics mechanisms, and event processing. In brief, the model incorporated in the simulation explicitly accounts for the following effects contributing to the observed resolution of a plastic scintillator: 1) variation in scintillation light-collection efficiency with interaction position in the scintillator, 2) statistical variation of energy deposition by a gamma scattering in the scintillator, 3) statistical variation in scintillation light emitted for a given energy deposition in a small region of the plastic, 4) statistical variation in actual light collection at a given PMT, given the mean value of lightcollection efficiency from a specified point in the scintillator, 5) statistical variation in the PMT photocathode response, i.e., number of photoelectrons produced by a given number of incident optical photons, and 6) statistical variation in PMT pulse-height amplification for a given number of electrons emitted from the photocathode.

Resolution effects that are not included in the simulation include 1) scintillation light "after-pulsing" arising from detection of long-decay-time fluorescence emission in the plastic (occurring in the course of gamma or background interactions before the current interaction of interest), 2) statistical variations in PMT dark current, and 3) statistical variations in line noise contributing to the analog PMT pulse integrated by the analog-to-digital converter (ADC). To empirically account for the latter three contributions, the simulation incorporates a final stage of gaussian "smearing" or "blurring" with a usersupplied value of the fractional standard deviation (or gaussian width parameter, sigma). Note that the contribution to the resolution induced by this final stage of smearing is expected to be roughly independent of the number and position of PMTs in the scintillator geometry. Thus, scintillator resolution variations arising from differing light-collection properties of various PMT configurations should be directly comparable with this final spectrum-blurring parameter held fixed.

Figure 7.1 displays representative output of the simulation for several point-like gamma sources in the single-PMT configuration described in Section 3. To match experimental conditions as closely as possible, the model incorporates a second (optically inactive) plastic scintillator slab located below the active scintillator, and the gamma source illuminates the system through a thin sheet of wood simulant. These sources of gamma-transmission scattering and backscattering have a relatively small effect on the results. As a general rule, the simulation predicts considerably better resolution for the Compton edge "peak" than is observed in the data, if no additional spectrum smearing is applied. The effect of the additional or "ad-hoc" smearing is demonstrated in Figure 7.2. To re-emphasize: all resolution-smearing effects of the model's light-collection-efficiency variations and various "information carrier" statistical effects (e.g., photon emission, PMT photoelectron emission, and PMT pulse amplification) are ostensibly taken into account in the unsmeared spectrum, and yet a final stage of pulse smearing is required to produce even a roughly realistic description of the data.

\footnotetext{
a The Geant4 code is available at http://wwwasd.web.cern.ch/wwwasd/geant4/geant4.html.
} 


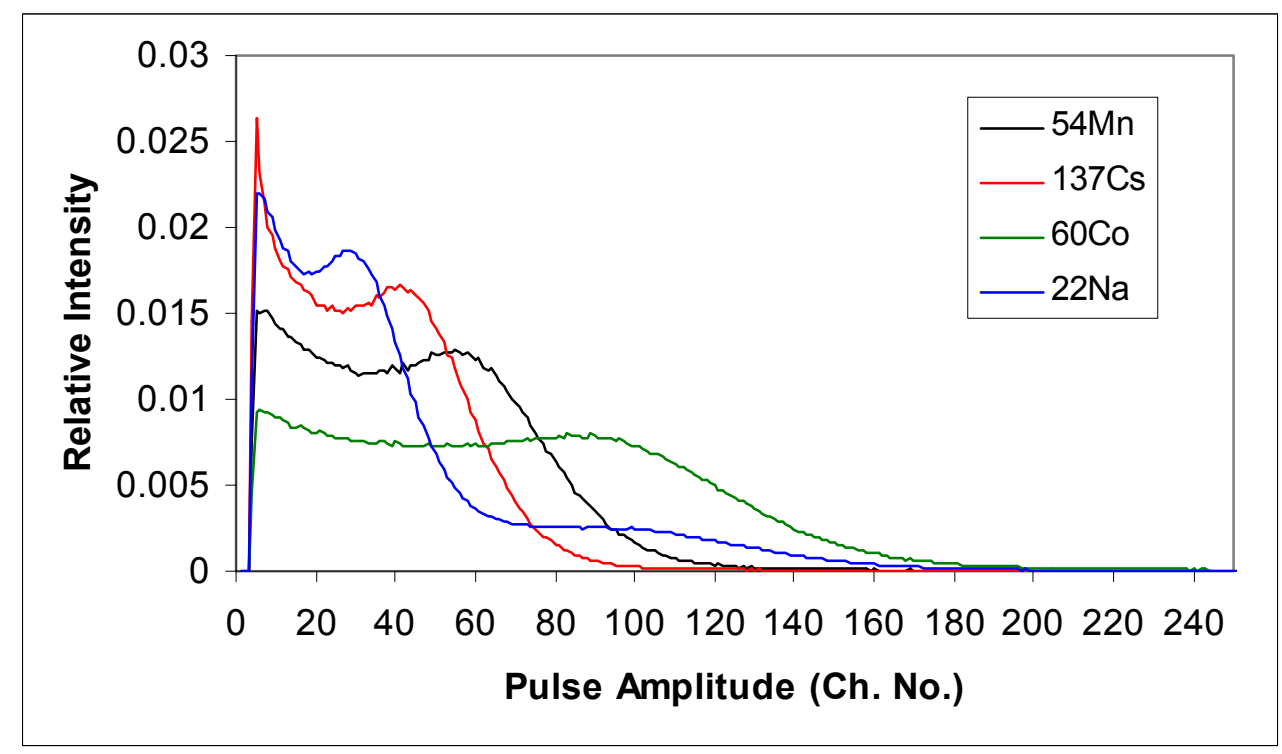

Figure 7.1. Simulation Output for Various Gamma Sources in Single-PMT Configuration. Spectra are normalized to unity. Simulation parameters for this multi-spectrum comparison have nominal values (i.e., not fine-tuned for comparison to data).

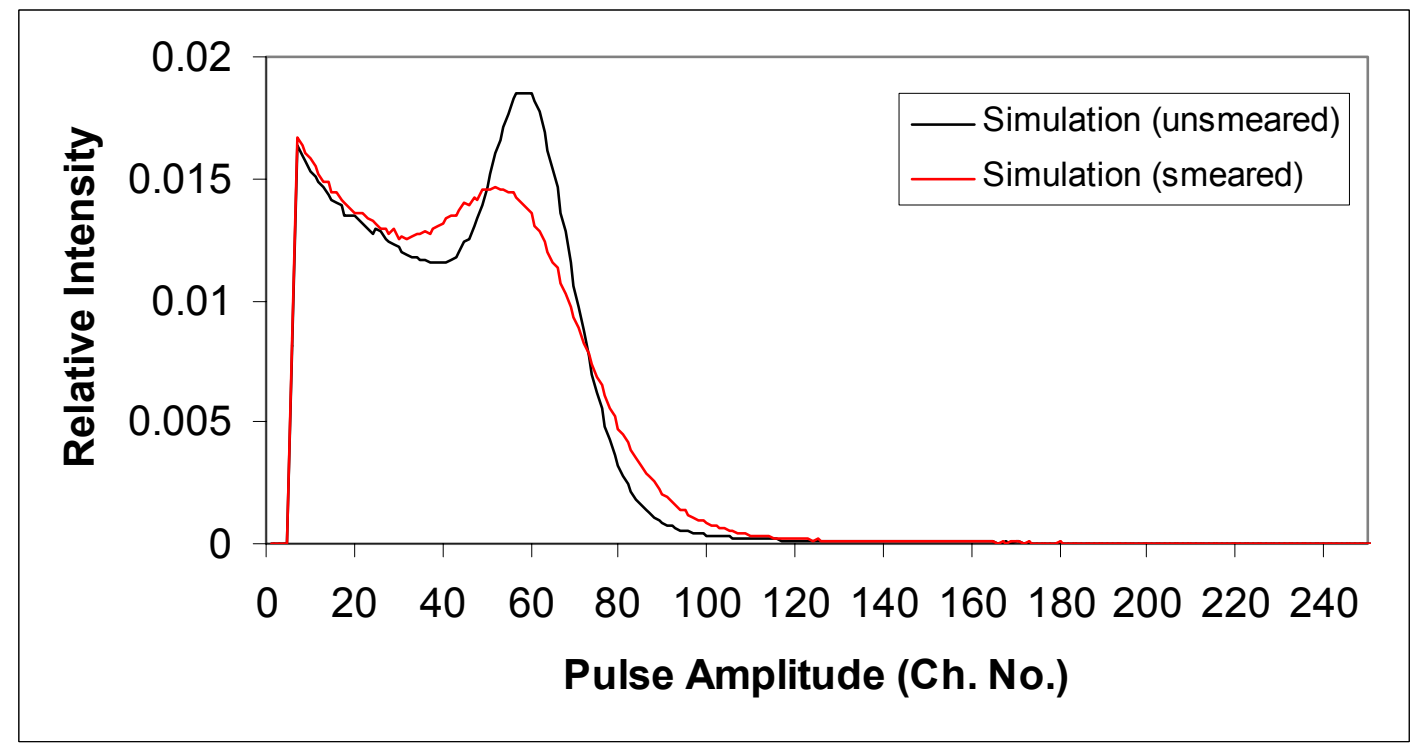

Figure 7.2. Effect of Final Pulse-Smearing Event Processing Stage on Simulation Output, ${ }^{54}$ Mn Gamma Source, One-PMT Configuration. Both spectra are normalized to unity.

At least two possible sources for this discrepancy can be postulated: 1) The lookup table of lightcollection efficiencies may be too coarsely spaced in position to accurately represent the variation observed in the data, especially at positions close to a PMT. The tables used for the preliminary investigations reported here incorporate only 10 divisions along the scintillator's $127 \mathrm{~cm}$ length, or almost 
$13 \mathrm{~cm}$ per division. The simulation's light-collection efficiency changes by roughly a factor of 3.6 over the last $13 \mathrm{~cm}$ before the PMT, and this rate of change may be too non-linear in the near-PMT region to permit accurate linear interpolation. An obvious solution to this model deficiency is to use a lightcollection-efficiency table with finer spacing, or to separate the problem into near-PMT and far-fromPMT regions, accounting for the two regions separately with different tables. These options are under investigation at the conclusion of FY 2003. 2) The light-attenuation length may be overestimated in the simulation. A nominal value of $380 \mathrm{~cm}$ for the optical attenuation length, quoted in Bicron/Saint Gobain technical specifications for the scintillant, is used in the simulation. Anecdotal evidence suggests that the attenuation length may be as low as $250 \mathrm{~cm}$ for specific batches of plastic. Lower light transmission would lead to lower light-collection efficiency, which would in turn yield poorer pulse-height resolution than observed in the data.

Figure 7.3 compares the simulation output to the experimental data for ${ }^{54} \mathrm{Mn}$ in the single-PMT configuration. Note that the simulation underpredicts the low-pulse-height response relative to the Compton edge and does a poor job of describing the shape of the distribution in the low-pulse-height region. This may be due to effects of the PMT dark current or the scintillator after-glow that are not adequately modeled by a simple resolution-smearing algorithm. As noted above, the simulation predicts better energy-deposition resolution than is observed in the data. One (indirect) metric of this resolution is the peak-to-valley ratio of the Compton edge. The peak-to-valley is expected to increase as the energydeposition resolution improves since events in the Compton edge will be more localized relative to the continuum at lower pulse height and should form a relatively higher "peak." This ratio is about 1.13 and 1.16 in the data and simulation, respectively.

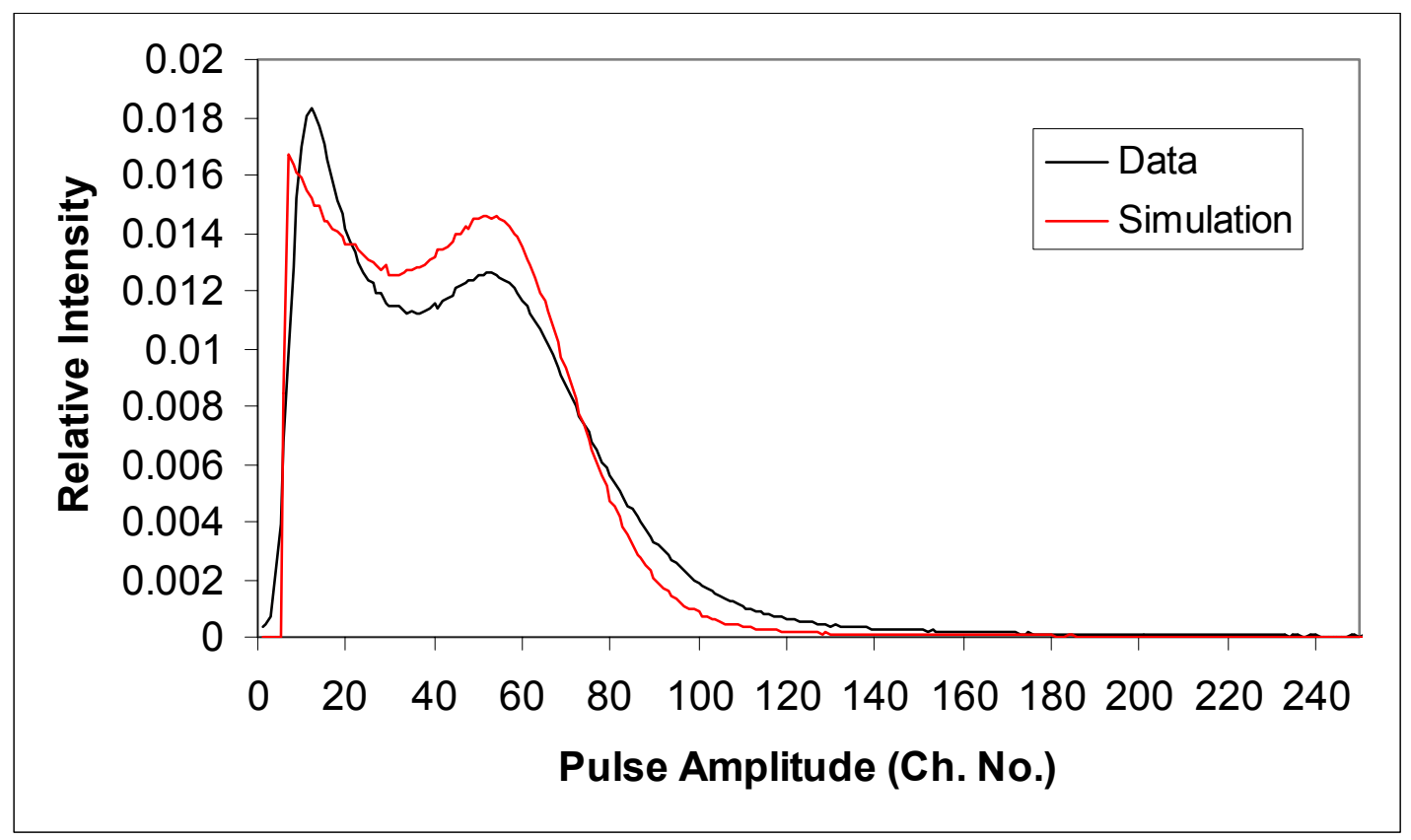

Figure 7.3. Comparison of Simulation to Experimental Data for ${ }^{54} \mathrm{Mn}$ Source in SinglePMT Scintillator Configuration. Both spectra are normalized to unity. 
An important point of comparison for the simulation is the effect of increased light-collection efficiency on the energy-deposition resolution. Figure 7.4 compares simulation to data for the configuration with one PMT at each end of the scintillator. In both data and simulation, the histograms used to form the (unity-normalized) distributions are incremented for events satisfying coincidence logic in which the output of each PMT is required to exceed a threshold. The summed output of the two PMTs is divided by two to maintain the same channel versus pulse-height scale as in the single-PMT case. The additional smearing factor applied to the simulation at the final stage of event processing has been held constant between the one-PMT and two-PMT configurations. Note that the reduction in low-pulse-height events afforded by the coincidence between PMTs has improved the agreement between simulation and data in this region, while at the Compton edge, the data now shows better resolution than the simulation. The Compton peak-to-valley ratios in this configuration are 1.48 and 1.34 for data and simulation, respectively, to be compared with 1.13 and 1.16 for data and simulation, respectively, in the one-PMT configuration. The resolution improvement in the data (according to this metric) is thus about $31 \%$, in contrast to only a $16 \%$ improvement in the simulation. The source of this discrepancy between predicted improvement and observed improvement is under investigation at the close of FY 2003. As noted above, an important contributing factor to this discrepancy may be that the position grid used to tabulate lightcollection efficiencies in the simulation is currently too coarse to accurately model the light-collection variation over the entire scintillator, especially near the PMT(s).

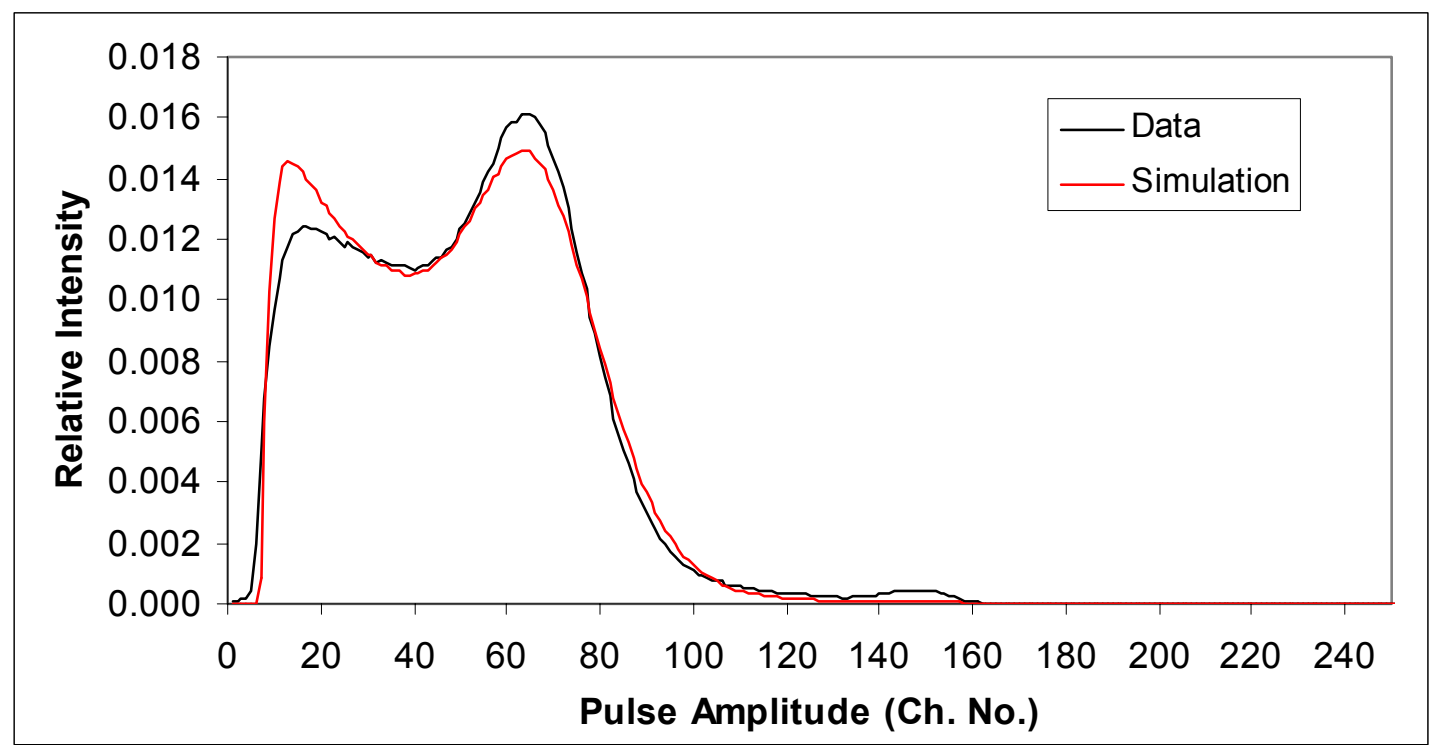

Figure 7.4. Comparison of Simulation to ${ }^{54} \mathrm{Mn}$ Source Data for Configuration with One PMT at each End. Both spectra are normalized to unity. 


\subsection{Conclusions and Outlook}

The experimental equipment and data-acquisition systems are fully functional to explore a variety of configurations involving multiple PMTs and one or two large scintillator slabs. In the second year of the ALPS project, we plan to measure pulse height and timing responses for the beta and gamma sources in a configuration with two PMTs on each end of the scintillator and again in a configuration with three PMTs on each end. Work on a timing-based position correction to pulse height is ongoing. Scintillator configurations with multiple PMTs on each end should allow position corrections to be applied in both the $\mathrm{x}$ and $\mathrm{y}$ dimensions. Data taken to date and simulation agree in indicating that multiple PMTs will give improved energy-deposition resolution because of the greater light-collection efficiency and reduced statistical uncertainty of the number of collected photons. In addition, $\mathrm{x}$ and y position corrections should give uniform light collection over the entire slab - again enhancing pulse-height resolution.

Further improvement in resolution should be achieved by adding a second scintillator slab in coincidence with the first. The second scintillator should allow capture of the scattered Compton gamma rays and perhaps be sufficient to capture all the energy of the incident gamma and thus give a "photopeak." The large capture efficiency and optimized resolution should allow better identification of gamma sources of interest in vehicle portal monitoring.

At this point in the project, we have demonstrated two important problems in identifying gamma sources using a large plastic scintillator and only one or two PMTs on the same side of the scintillator slab. These problems are inefficient light collection and non-uniform response as a function of location of the interaction. A configuration with one PMT on each end of the slab shows greatly improved resolution and permits corrections to the light-output response based on the location of the interaction. This configuration can only give significant improvements for events in direct line-of-sight between the two phototubes. Multiple PMTs on each end should allow corrections to be applied for events occurring anywhere in the $x-y$ plane of the scintillator and thus provide a uniform response. It is anticipated that optimizing the energy-deposition resolution in plastic scintillator will permit significant improvements in the capability to identify unknown gamma sources using a large-area plastic sensor. The extent to which improved resolution enhances source-identification capability will be quantified by a combination of ALPS data, simulation, and statistical analysis in the second year of the project. 


\subsection{References}

Agostinelli, S. et al. "Geant4 -- A Simulation Toolkit." Nuclear Instruments and Methods A 506 (2003), 250-303.

Clark D. 1974. "The Intrinsic Scintillation Efficiency of Plastic Scintillators for ${ }^{60}$ Co Gamma Excitation." Nuclear Instruments and Methods 117:295-303.

Jordan DV, BD Geelhood, PL Reeder, DL Stephens, RC Craig, and JI McIntyre. 2003. Progress Report on the Advanced Large-Area Plastic Scintillators (ALPS) Project. PNNL-14283, Pacific Northwest National Laboratory, Richland, WA. 


\section{Appendix A}

\section{Details of the ALPS Simulation}




\section{Appendix A: Details of the ALPS Simulation}

This appendix furnishes a more complete description of the ALPS Geant4 simulation outlined briefly in Section 4. The current version of the simulation includes the following features:

- a flexible user interface for specifying scintillator geometry, with the option for specifying two scintillator shapes (parallelepiped and beveled-corner) and multiple PMTs on a scintillator

- a variety of ionizing radiation and optical photon source-term options

- two methods for computing optical photon transmission in the scintillator, explicit ray tracking, or light-collection lookup table

- a simple numerical model of the photoelectron ejection and stage-amplification processing of optical photons incident on the PMT photocathode.

\section{A.1 Simulation Geometry}

To accommodate a variety of scintillator and PMT configurations tested during the course of the ALPS project, the simulation's geometry-specification interface was designed for reasonable flexibility and ease of use in specifying, for example, PMT and light-guide positions. The current version of the simulation permits the user to define a single scintillator slab with up to 10 PMTs (with or without accompanying light-guides) attached to the slab. The plastic scintillant slab can be one of two shapes: 1) parallelepiped (i.e., box-shaped) of user-specified length, width, and thickness, or 2) parallelepiped with beveled corners (beveled faces oriented at 45 degrees with respect to the adjacent scintillator edges), for which the user must supply the dimensions of the containing box shape's length, width, and thickness as well as the length of the beveled edges. The slab of plastic scintillant is suspended in a cubical, air-filled "laboratory" volume of user-specified edge length, with the center of the slab located at the origin of the laboratory volume's Cartesian coordinate system. The z-axis in the laboratory coordinate system coincides with the long axis of a typical large-area scintillator, with the x-axis lying along the "width" direction and the y-axis lying along the "thickness" direction. For the purpose of modeling ionizingradiation interactions, the plastic scintillant material filling the slab volume is defined by its chemical composition, $\mathrm{C}_{9} \mathrm{H}_{10}$, and density, $1.032 \mathrm{~g} / \mathrm{cm}^{3}$. Optical properties of the scintillant are specified by the user-supplied index of refraction and optical attenuation length, and a choice of several optical coatings is available, including 1) direct scintillant-to-air coupling, 2) specular (i.e., smooth metallic foil) reflecting coat with user-specified reflectivity, with or without an intervening air gap between the foil and the scintillant, or 3) diffusely reflecting coat, again with user-specified reflectivity.

The simulation's PMTs consist of simple boxes or cylinders, filled with material of the same composition and density as the scintillator slab. Dimensions and positions of the PMT volumes are specified individually for each tube in the simulation's input file. To minimize the possibility of incorrect positioning of the PMT tube at the scintillator edge (e.g., leaving a gap between the PMT entrance and the scintillator volume, or overlapping the PMT and the scintillator), the code provides the option for "autopositioning" of the PMT volume against the edge of the scintillator. In the case of a parallelepiped, or standard-shaped, scintillator, this option amounts to automatic determination of the PMT position along the long-axis (i.e., z-direction) of the system so that the edges of PMT and scintillator volumes align 
properly. In the case of a beveled-corner scintillator, PMTs may be auto-positioned along the center of each straight scintillator edge or at the center of a beveled corner, with correct rotations taken into account for the latter. The index of refraction of the PMT material is hard-wired in the code as 1.581, a typical value for Bicron/St. Gobain BC-408 scintillant. Because the index of refraction and chemical properties of the simulation's PMT volumes match nominal values for BC-408 scintillant, the PMTs act only as sensitive regions for detecting the arrival of optical photons and signaling the tracking framework to terminate the current photon's history. Thus, effects arising from photon reflection and refraction at the surface of the PMT, or angle-of-incidence dependence in the PMT photon acceptance, are neglected in the current simulation. (Studies of the effect of angle-of-incidence restrictions on the photon acceptance were reported in Jordan et al. (2003).

\section{A.2 Source-Term Specification}

The input framework for specification of the simulation's source term has been designed for maximum flexibility in modeling a variety of source types, spatial positions, angular-emission distributions, and energy distributions. Three general source particle types are supported: optical photons, gamma rays, and electrons. The optical photon source type is typically used for mapping the light-collection-efficiency properties of a given scintillator + PMT configuration; in this mode, the source points for light generation are distributed within the scintillator volume. Gamma and electron source types are used for modeling the energy deposition (and attendant light-collection) response of the scintillator to an external source of ionizing radiation. Two options for distribution of source position are supported: fixed position and uniform distribution within a user-specified box volume. The fixed-position mode is the more commonly used of the two, but the uniform distribution within a box volume can be used for conveniently modeling the volume-averaged light-collection efficiency over an entire scintillator. The angular distribution of source-particle trajectories can be specified as one of three options: 1) fixed direction (user provides components of the direction vector), 2) isotropic distribution, or 3) isotropic distribution over a limited portion of $4 \pi$ solid angle, from polar angle 0 to a user-specified maximum, $\theta_{\max }$. The third option can be used for efficient illumination of the scintillator surface by a gamma point source suspended above the scintillator. Finally, several energy distributions are supported for gamma and electron source types. (In the case of optical photons, the simulation currently supports only monoenergetic photons, with the photon energy hard-coded at $3 \mathrm{eV}$.) The user can supply either a single energy, a lookup table to generate a discrete energy distribution (e.g., for a set of gammas emitted by a given radionuclide), or a lookup table for a continuous energy distribution (e.g., for the electron energy spectrum of a beta-emitter.) In the case of energy sampling from discrete or continuous distributions, the generated energy is histogrammed and output at the end of the simulation as a diagnostic.

\section{A.3 Simulation Modes}

The simulation features several distinct event-processing modes in support of a strategy for scintillator + PMT modeling that includes the following two stages: 1) Mapping of the light-collection response of the system as a function of optical-photon-emission position within the scintillator volume, independent of the source of the scintillation photons in the sensor. 2) Convolution of the energy-deposition response of the scintillator to an external source of ionizing radiation with the light-collection properties of the scintillator + PMT configuration. As explained in the body of this report, the code can be run in a mode where the interaction of incident ionizing radiation (e.g., gammas or betas from a point source) and the attendant transport of all scintillation photons emitted due to the energy deposition from the incident 
radiation are simulated on an event-by-event basis. However, this operating mode is relatively inefficient and CPU-intensive, since many optical photons (of order 1,000 to 10,000) can be generated for every incident-ionizing-radiation quantum. On a "typical" PC with CPU speed on the order of $1 \mathrm{GHz}$, this "explicit ray tracing" operating mode can require several hours to simulate only a few thousand input radiation quanta.

A more practical approach to achieving the convolution of the scintillator energy deposition and lightcollection responses is to break the simulation process into two stages. In the first stage, the lightcollection properties of the scintillator are mapped as a function of optical-photon-emission position. The results of this simulation stage take the form of a lookup table in three spatial dimensions, listing the light-collection efficiency for each PMT in the system as a function of position within the scintillator. In the second simulation stage, the interaction of incident-ionizing radiation with the scintillant is modeled, with step-wise energy deposition along the track of the incident particle (and any secondary particles, such as electrons Compton-scattered by a gamma). The energy deposition at each step in the tracking through the scintillant is converted into a mean number of scintillation photons, with scintillation efficiency specified by the user. A typical value for this scintillation efficiency is 10,000 optical photons per $\mathrm{MeV}$ of energy deposition. Interpolation on the lookup table for light-collection efficiency then determines the number of photons reaching each PMT from the current tracking step. The number of photons reaching each PMT along the entire scintillator track of the incident ionizing radiation quantum (and any of its secondaries) is accumulated, and they form the input for modeling the response of each PMT for that event. Note that the time savings in this "Fast Optics" mode arises from the use of a lookup table to determine the fraction of scintillation photons reaching each PMT from a given point along the ionizing-radiation quantum's path in the scintillator, rather than tracking each individual optical photon. The use of the lookup-table optics method is justified to the extent that 1) there is no correlation between the incident radiation quantum's position and velocity and the direction of emission of the optical photons (i.e., isotropic emission throughout the scintillator), 2) the light-collection properties of the scintillator vary smoothly and relatively slowly on the scale of the spatial grid used to form the lookup table and 3) variations of the light-collection acceptance of the PMT as a function of photon position and angle of incidence at the photocathode are neglected.

The algorithm for computing the light incident on each PMT in this "Fast Optics" mode may be specified in greater detail as follows:

- For each simulation event, an incident radiation quantum (e.g., gamma or beta) is propagated through the scintillator medium. The incident quantum, or its secondaries, deposit energy dE at position $(\mathrm{x}, \mathrm{y}, \mathrm{z})$ for each tracking step within the scintillator volume.

- For each tracking step, the energy deposition $\mathrm{dE}$ in that step is used to calculate the mean number of scintillation photons, $\mathrm{N}_{1}$, generated at that point in the scintillator. $\mathrm{N}_{1}$ is obtained by multiplying $\mathrm{dE}$ by the scintillation efficiency, specified in number of photons per $\mathrm{MeV}$. The actual number (as opposed to the distribution mean number) of photons generated at this point, $\mathrm{N}_{2}$, is then sampled from either a Gaussian distribution of mean $\mathrm{N}_{1}$ and standard deviation $\mathrm{N}_{1}{ }^{1 / 2}$, if $\mathrm{N}_{1}$ is greater than or equal to 10 photons, or a Poisson distribution of mean $\mathrm{N}_{1}$ if $\mathrm{N}_{1}$ is less than 10 .

- The mean number of photons, $\mathrm{N}_{3}$, reaching each PMT is determined from $\mathrm{N}_{2}$ by determining the light-collection efficiency, $\varepsilon$, for each PMT at point $(\mathrm{x}, \mathrm{y}, \mathrm{z})$ from a lookup table: $\mathrm{N}_{3}=\varepsilon \mathrm{N}_{2}$. The actual 
number of photons reaching each PMT, $\mathrm{N}_{4}$, is then determined by sampling from a Gaussian distribution of mean $\mathrm{N}_{3}\left(\mathrm{~N}_{3} \geq 10\right)$ or a Poisson distribution of mean $\mathrm{N}_{3}\left(\mathrm{~N}_{3}<10\right)$. The standard deviation for the Gaussian sampling case is modified from the usual "counting statistics" value of $\mathrm{N}_{3}{ }^{1 / 2}$ by a factor of $(1-\varepsilon)^{1 / 2}$ to account for the fact that the probability for an individual optical photon to reach a PMT is not necessarily small compared to 1 , especially for positions close to a PMT. Thus a binomial distribution characterized by "success" and "failure" probabilities $(P, Q)=(\varepsilon, 1-\varepsilon)$ should in principle be sampled $\mathrm{N}_{3}$ times to determine how many of the $\mathrm{N}_{3}$ optical photons reach a given PMT. In practice, a reasonable approximation to the resulting distribution can be obtained simply by sampling a Gaussian distribution of mean $\mathrm{N}_{3}$ and standard deviation $\left[\mathrm{N}_{3}(1-\varepsilon)\right]^{1 / 2}$.

- For each PMT, the total number of optical photons reaching that PMT (given by $\mathrm{N}_{4}$ in the paragraph above) is summed over the entire tracking history of the current event's incident-radiation quantum. The current version of the simulation does not yet account for the arrival time of the optical photons, but this information is available from the particle-tracking framework and will be incorporated in a future version of the code. Adding timing information to the simulation will enable modeling investigation of various TDC position-identification schemes for implementing a pulse-height correction.

\section{A.4 PMT Light-Amplification Model}

Event processing for each incident radiation quantum tracking history culminates in the accumulation of the number of optical photons incident on each PMT in the model's scintillator + PMT configuration. At this point, a simple numerical model for representing the photoelectron (PE) generation and pulseamplification stages of the PMT is applied to the input optical photons to generate an output "signal" from the PMT. The steps in the PMT signal-generation algorithm are as follows:

- Let the total number of optical photons incident on the PMT (i.e., the sum over all tracking steps of $\mathrm{N}_{4}$ in A.3) be $\mathrm{N}_{\mathrm{op}}$. The number of PEs generated due to the interaction of these photons with the PMT photocathode, of quantum efficiency $(\mathrm{QE})$, is determined by sampling from a Gaussian of mean $\mathrm{N}_{5}=$ $\left(\mathrm{QE} \mathrm{N} \mathrm{N}_{\mathrm{op}}\right)$ and standard deviation $\left[\mathrm{N}_{5}(1-\mathrm{QE})\right]^{1 / 2}$, for $\mathrm{N}_{5} \geq 10$, or from a Poisson distribution of mean $\mathrm{N}_{5}$, for $\mathrm{N}_{5}<10$. As in the case of determining the number of optical photons reaching the PMT, the reason for the factor of $(1-\mathrm{QE})^{1 / 2}$ in the expression for the standard deviation in the Gaussian sampling case is to account for the sampling of each individual photon's interaction with the photocathode - a process governed by the binomial distribution with "success" and "failure" probabilities $(\mathrm{P}, \mathrm{Q})=(\mathrm{QE}, 1-\mathrm{QE})$.

- The effect of the PMT pulse-amplification stages on the initial set of $\mathrm{N}_{5}$ photoelectrons is modeled by applying $\mathrm{M}$ user-specified stage-multiplication factors $\mathrm{F}_{1}, \mathrm{~F}_{2}, \ldots, \mathrm{F}_{\mathrm{M}}$. The overall multiplication factor, $\mathrm{F}$, is given by the product of the M-stage multiplication factors. The number of output PMT electrons, $\mathrm{N}_{\text {out }}$, is sampled from a Gaussian distribution of mean $\mathrm{N}_{6}=\mathrm{F} \mathrm{N}_{5}$. The standard deviation of the distribution is determined using a generalization to multiple PEs and (possibly) distinct stage gains of a description of the variance for single-PE PMT multiplication obtained from Knoll (2000), Equation (9.4). Let $\sigma$ be the standard deviation in the distribution of output electrons after $M$ multiplication stages. Then $\sigma=\left(\mathrm{GN}_{6}\right)^{1 / 2}$, where the variance factor $\mathrm{G}$ is given by 


$$
G=F\left(\frac{1}{F_{1}}+\frac{1}{F_{1} F_{2}}+\frac{1}{F_{1} F_{2} F_{3}}+\cdots+\frac{1}{F_{1} F_{2} \cdots F_{M}}\right)
$$

- Finally, the number of output electrons $\mathrm{N}_{\text {out }}$ is scaled by an empirical, user-supplied factor to convert the PMT output "pulse" to a quantity that can be histogrammed and compared directly to experimental ADC spectra. This scale factor represents the effect of pulse amplification and digitization in the scintillator pulse-processing electronics and any additional scale factor(s) that may be applied to the ADC output in the data-acquisition software before forming the final pulse-height histogram.

To mimic the effect of discriminator thresholds for logic-level pulse formation and generation of the experimental data-acquisition trigger, which may in general require the coincidence of pulses from several PMTs, the simulation code allows the user to provide a "pulse discrimination threshold" for the output of each PMT, and a list of PMTs that must have signals above this threshold to satisfy the coincidence logic. This coincidence PMT signal condition is used to gate the incrementing of histograms of the individual and summed PMT signals in units compatible with the experimental pulse-height spectra.

\section{A.5 Simulation Output}

In the mapping mode for optical-photon light efficiency, the simulation output consists of a set of counters, one for each PMT, representing the number of events in which an optical photon reached the PMT in question. The light-collection efficiency for a PMT is then calculated from the simulation output simply by dividing that PMT's hit counter by the number of generated photons.

In the "Fast Optics" mode, in which an external radiation source illuminates the scintillator, the basic simulation output consists of a set of histograms of the following quantities:

- energy of generated incident radiation quantum (if not in mono-energetic source mode)

- energy deposition in the scintillator

- number of scintillation photons generated, mean and distribution-sampled

- ADC spectra for each PMT and sum over PMTs

- coincidence-gated ADC spectra for each PMT and sum over PMTs.

A number of diagnostic histograms are also output in Fast Optics mode for a single PMT only:

- number of optical photons reaching PMT \#1, mean and distribution-sampled

- number of photoelectrons generated in PMT \#1, mean and distribution-sampled

- number of electrons at exit multiplication stage of PMT \#1, mean and distribution-sampled.

In practice, adjustment of the empirical scale factor to convert "PMT output" to an ADC-spectrum compatible quantity is accomplished by noting the spectrum channel number of some prominent feature in the experimental data spectrum (e.g., the Compton edge for a given gamma source) and calculating the ratio of this channel number to the analogous spectrum feature in the simulation's PMT \#1 output pulse spectrum. 


\section{A.6 References}

Knoll G. 2000. Radiation Detection and Measurement. Third Edition, John Wiley \& Sons, New York, NY.

Jordan DV, BD Geelhood, PL Reeder, DL Stephens, RC Craig, and JI McIntyre. 2003. Progress Report on the Advanced Large-Area Plastic Scintillators (ALPS) Project. PNNL-14283, Pacific Northwest National Laboratory, Richland, WA. 
Appendix B

Measurements on 30-cm $\times 30-\mathrm{cm}$ Beveled Corner Scintillator 


\section{Appendix B: Measurements on 30-cm $\times$ 30-cm Beveled Corner Scintillator}

In preparation for work with the large-area scintillator slabs that represent the primary focus of the ALPS project, a set of experiments was performed on a $30 \times 30 \times 6 \mathrm{~cm}^{3}$ plastic scintillator viewed by four PMTs located on the beveled corners. A ${ }^{90} \mathrm{Sr}$ beta source was located in the recess of an aluminum cylinder having an $\approx 1$-cm hole through $\approx 1$-cm thickness of aluminum. Pulse-height spectra were obtained for each PMT and for the sum of all four PMTs. Source locations were at various intervals within one quadrant of the scintillator. Spectra were measured at three locations along the diagonal pointing toward PMT 3, at four locations along the midline pointing toward the midpoint between PMTs 2 and 3, at four locations on a line between the diagonal and the midline, and at the center of the scintillator. All data were obtained with the requirement that all four PMTs recorded a coincident event. This coincidence requirement means that some events at low pulse heights were lost if one PMT did not see a pulse above its discriminator.

Because the system has 4-fold symmetry, the spectra from PMT 3 obtained from points located on the diagonal between the center and PMT 3 can be combined with the spectra from PMT 1 taken simultaneously to give a set of spectra representing the pulse-height response along the entire diagonal from PMT 3 to PMT 1. Likewise, the pulse-height spectra from PMT 3 and PMT 4 were used to give a complete set of responses along the midline as a function of the distance from the midpoint of one edge to the midpoint of the opposite edge.

Figure B1 gives pulse-height spectra from PMT 1 for four locations on the diagonal between PMT 1 and PMT 3. The pulse-height spectrum in PMT 1 is equivalent to the pulse-height spectrum in PMT 3 if the source were $34 \mathrm{~cm}$ from PMT 3. Similarly, the pulse-height spectrum in PMT 1 is equivalent to the pulse-height spectrum in PMT 3 if the source were $25.4 \mathrm{~cm}$ from PMT 3. The pulse-height spectra in PMT 3 are shown in Figure B2 for the same locations as in Figure B1. The general conclusion is that as the source is moved farther from PMT 3, the spectrum shifts to lower pulse heights, but that there is relatively little change when the source is at the midpoint and farther away. 
90Sr Response on Diagonal - PMT1

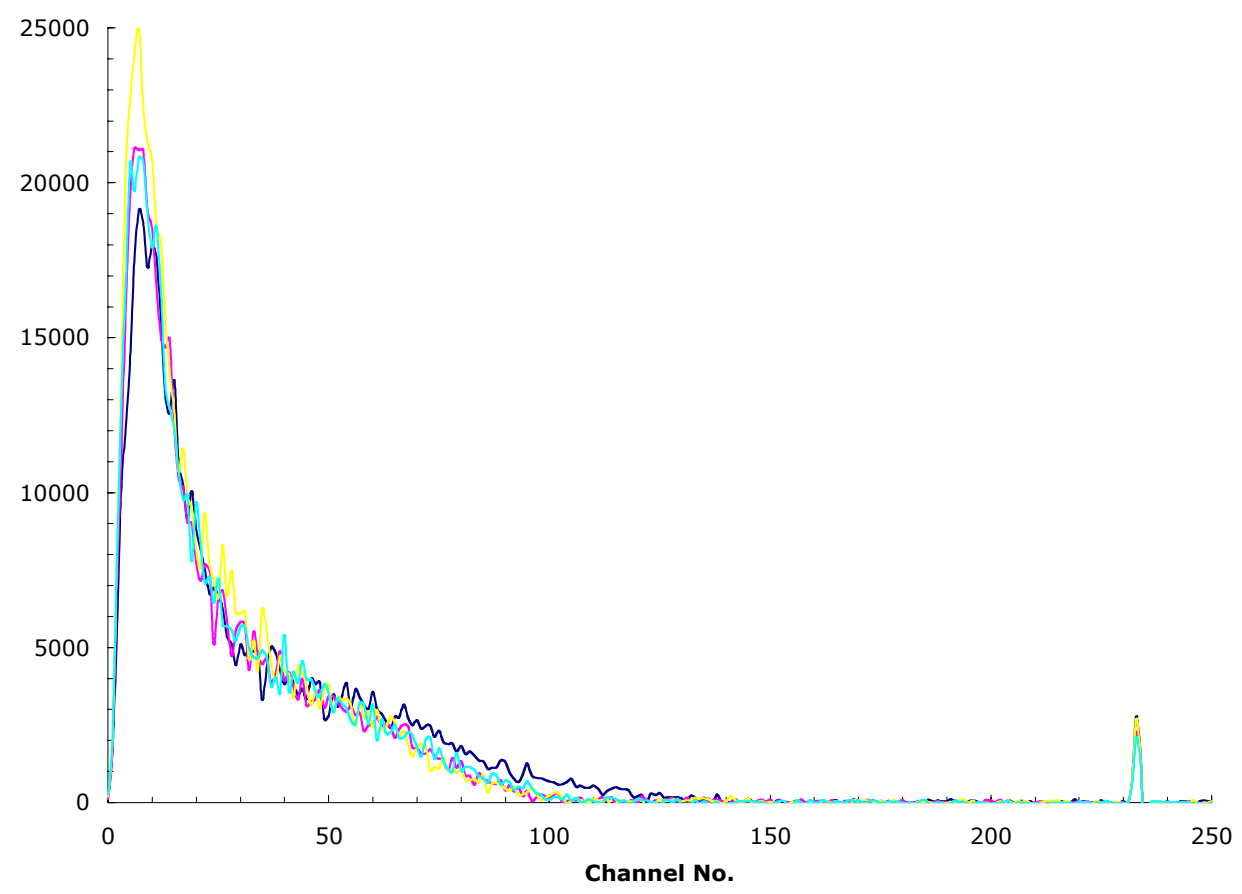

Figure B1. Pulse Height Spectra Along Diagonal for PMT 1

90Sr Response on Diagonal - PMT3

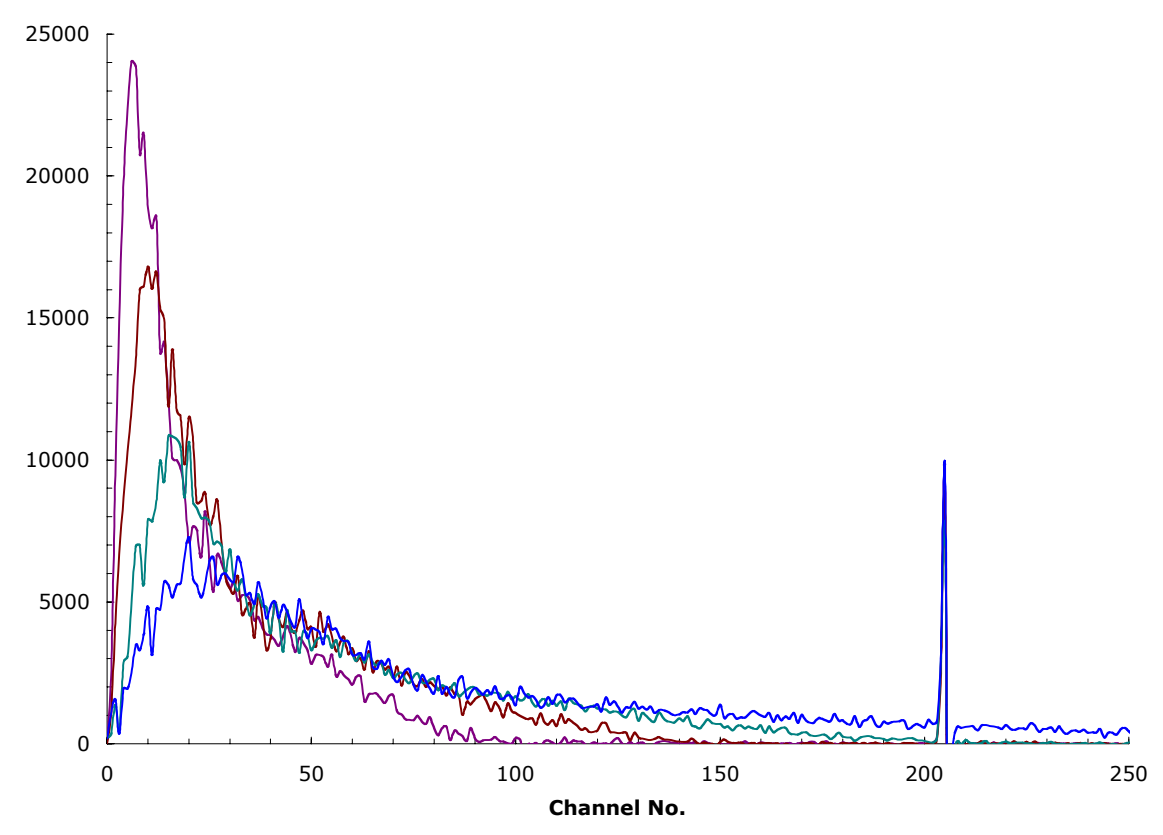

Figure B2. Pulse Height Spectra Along Diagonal for PMT 3 
Figure B3 shows pulse-height spectra for the sum of all four PMTs at the four locations along the diagonal between PMT1 and PMT 3. The pulse-height spectrum taken closest to PMT 3 has fewer lowenergy events and more high-energy events than the spectra taken farther away. This is consistent with the previous results in that greater pulse heights are observed as the location gets close to one PMT but without much loss in pulse height from the other three PMTs.

\section{Sr Response on Diagonal - Sum 4 PMTs}

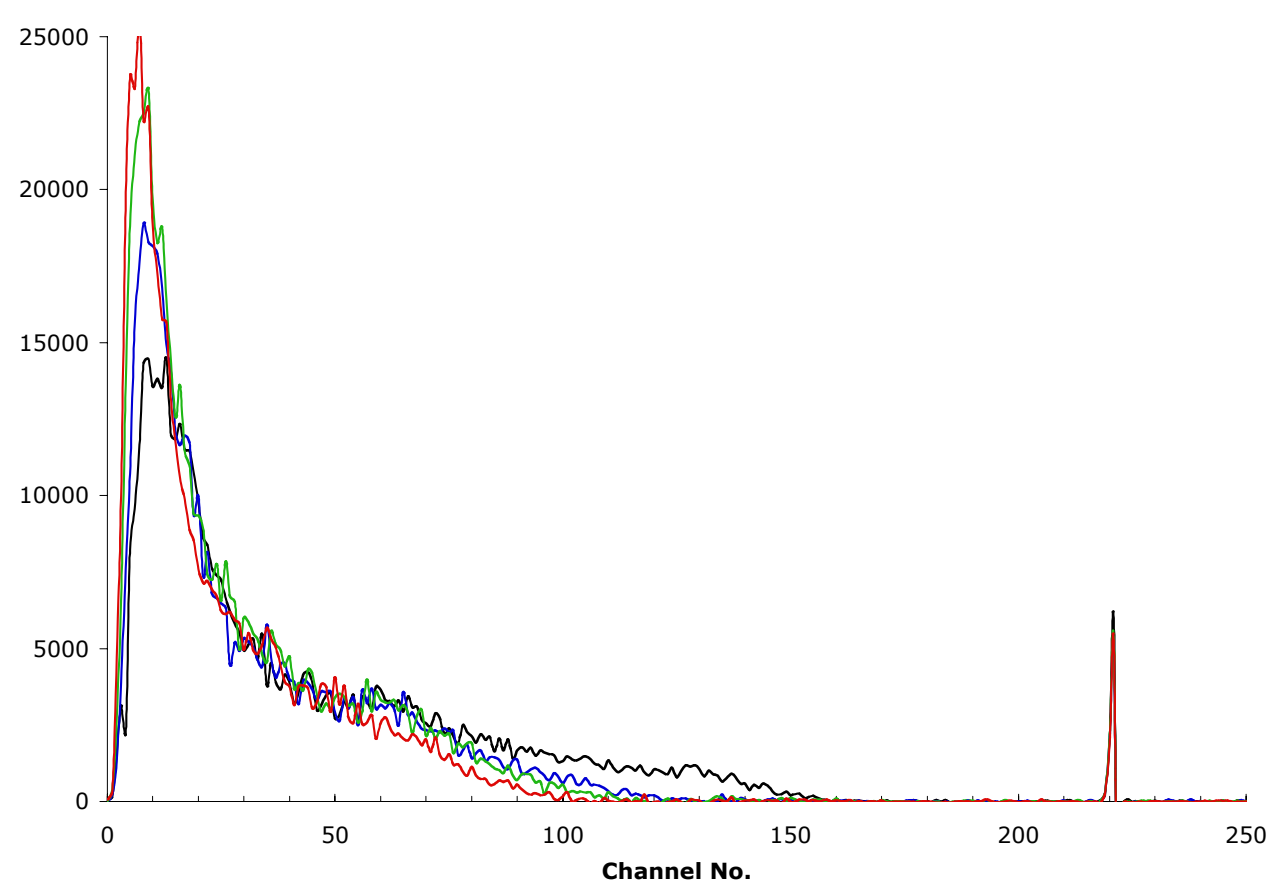

Figure B3. Pulse Height Spectra Along Diagonal for Sum of Four PMTs

The results for pulse-height spectra taken along the midline differ from the diagonal results in that there is relatively little change in pulse-height spectra as a function of distance going from one edge to the opposite edge. Results for PMT 4 are shown in Figure B4, and results for PMT 3 are shown in Figure B5. The pulse-height spectra from the sum of all four PMTs are shown in Figure B6. The sum spectra are consistent with the spectra for individual PMTs in that there is little change as a function of position along the midline. The general conclusion is that the pulse-height spectra are rather uniform for both the individual PMTs and for the sum of all four PMTs except when the source is located close to one of the PMTs - in which case, the spectrum shifts to higher pulse heights. The count rate is essentially constant regardless of the source location. This conclusion is valid only for this experiment where only 4-fold coincidence events were accepted. 


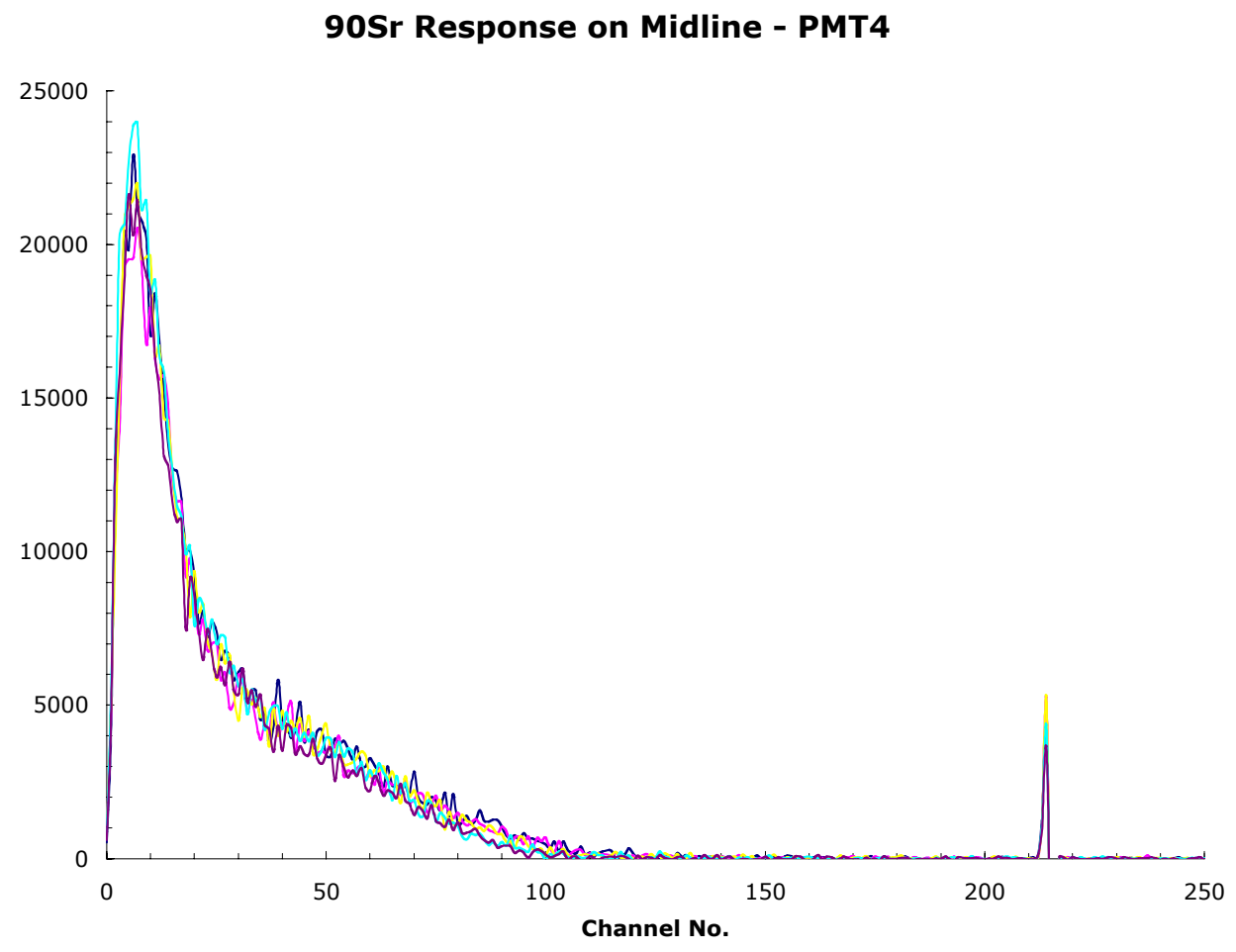

Figure B4. Pulse-Height Spectra Along Midline for PMT 4

90Sr Response on Midline - PMT3

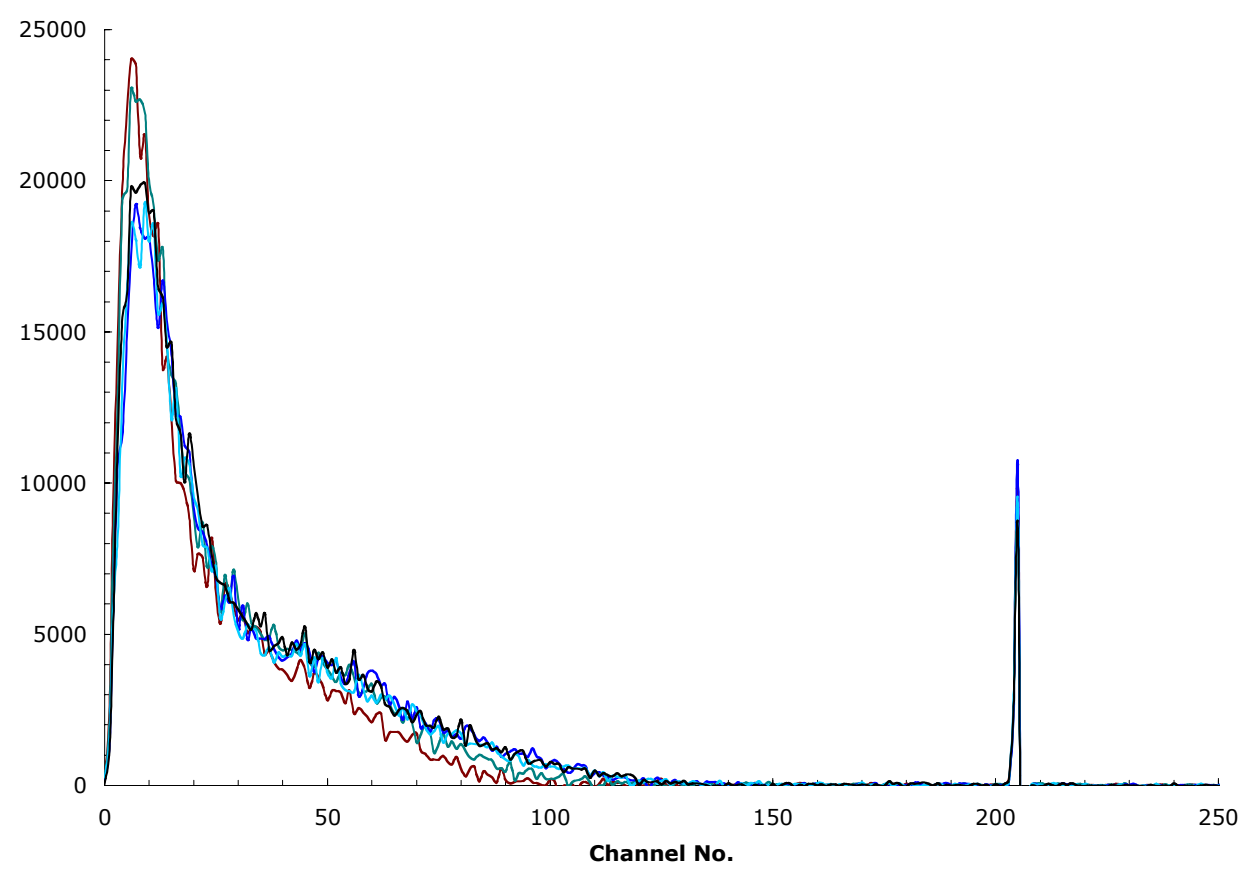

Figure B5. Pulse-Height Spectra Along Midline for PMT 3 
90Sr Response on Midline - SUM PMTs 1234

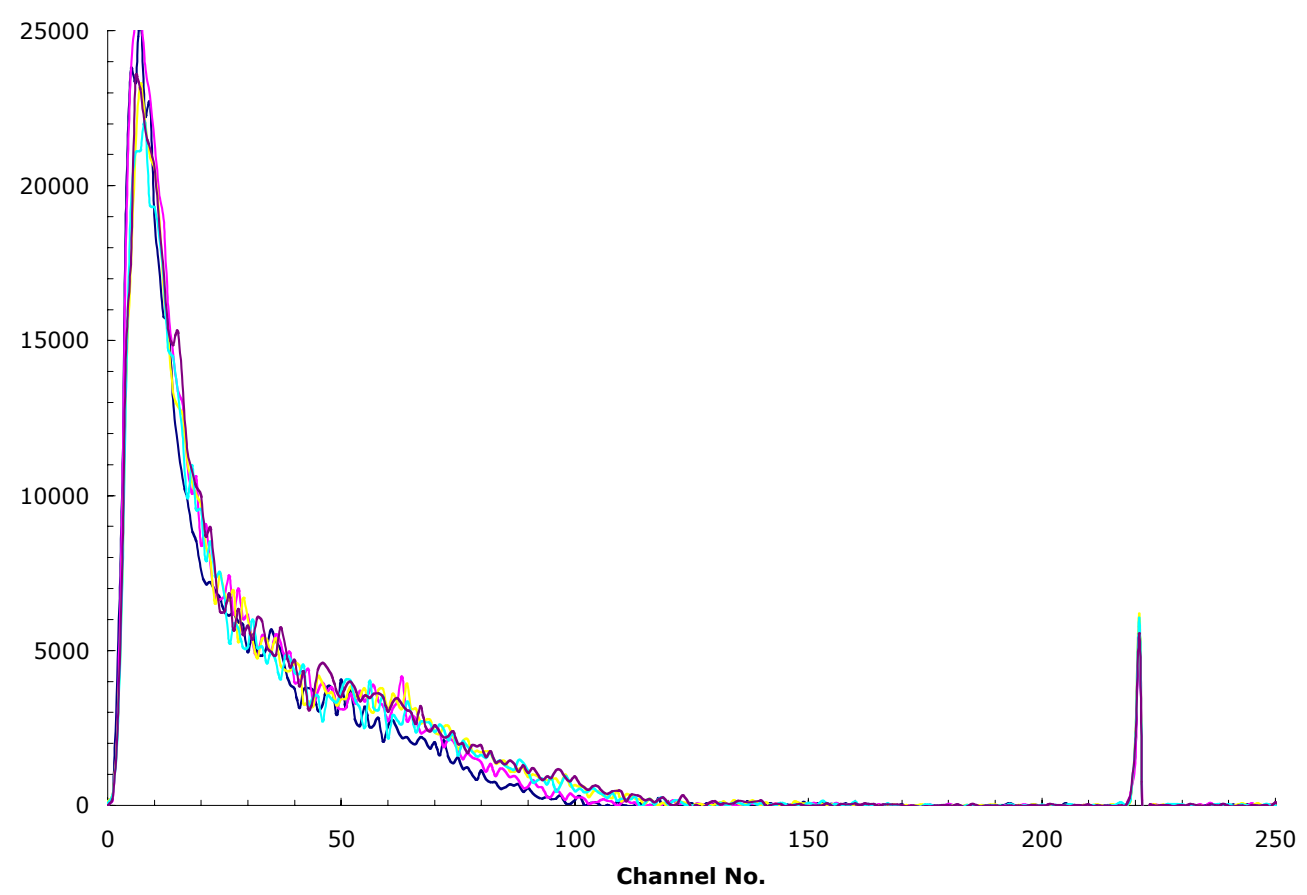

Figure B6. Pulse-Height Spectra Along Midline for Sum of Four PMTs 


\section{Distribution}

No. of

Copies

\section{OFFSITE}

1 David Spears, NA-22 (2 copies)

U.S. Department of Energy 1000 Independence Ave., S.W.

Washington, D.C. 20585-0420

1 Michael O'Connell, NA-22 (2 copies)

U.S. Department of Energy

1000 Independence Ave., S.W.

Washington, D.C. 20585-0420
No. of

Copies

\section{ONSITE}

12 Pacific Northwest National Laboratory

D. V. Jordan (2)

P8-20

P. L. Reeder

P8-50

R. A. Craig

P8-50

G. B. Dudder

K8-29

B. D. Geelhood

P8-01

R. R. Hansen

K8-03

R. T. Kouzes

P8-20

D. L. Stephens

P8-20

G. A. Warren

P8-20

Technical Report Files (2)

Distr. 1 